\title{
Aspects of flavour and supersymmetry in F-theory GUTs
}

\author{
Joseph P. Conlon ${ }^{a}$ and Eran Palti ${ }^{b}$ \\ ${ }^{a}$ Rudolf Peierls Centre for Theoretical Physics, \\ 1 Keble Road, Oxford OX1 3NP, U.K. \\ ${ }^{b}$ Centre de Physique Théorique, Ecole Polytechnique, CNRS, \\ 91128 Palaiseau, France \\ E-mail: j.conlon1@physics.ox.ac.uk, palti@cpht.polytechnique.fr
}

\begin{abstract}
We study the constraints of supersymmetry on flavour in recently proposed models of F-theory GUTs. We relate the topologically twisted theory to the canonical presentation of eight-dimensional super Yang-Mills and provide a dictionary between the two. We describe the constraints on Yukawa couplings implied by holomorphy of the superpotential in the effective 4-dimensional supergravity theory, including the scaling with $\alpha_{\text {GUT }}$. Taking D-terms into account we solve explicitly to second order for wavefunctions and Yukawas due to metric and flux perturbations and find a rank-one Yukawa matrix with no subleading corrections.
\end{abstract}

KEYwords: F-Theory, Intersecting branes models, Quark Masses and SM Parameters

ARXIV EPRINT: 0910.2413 


\section{Contents}

1 Introduction 1

2 Local models of intersecting branes in F-theory 2

2.1 Effective theory for intersecting 7-branes 2

2.2 Zero modes and Yukawa couplings 4

3 Relationship to super Yang-Mills $\quad 6$

$\begin{array}{ll}3.1 \text { Equations of motion } & 7\end{array}$

$\begin{array}{lll}3.2 & \text { Yukawa couplings } & 12\end{array}$

$\begin{array}{lll}3.3 & \text { Holomorphy constraints on the effective theory } & 16\end{array}$

4 Wavefunctions and Yukawas with background fluxes $\quad 17$

$\begin{array}{lll}4.1 & \text { Flux solutions to D-terms } & 17\end{array}$

$\begin{array}{lll}4.2 & \text { Matter wavefunctions } & 19\end{array}$

$\begin{array}{llr}4.3 & \text { Higgs wavefunctions } & 20\end{array}$

4.4 Yukawa couplings 21

5 Summary and discussion $\quad 23$

\section{Introduction}

The flavour mass hierarchies of the standard model are a tantalising hint towards deeper and more fundamental structures in theoretical physics. As a candidate fundamental theory string theory is a natural arena in which to study this topic. There has been recent interest in F-theory model building, in part motivated by the ability to obtain an $\mathcal{O}(1)$ top Yukawa coupling. These models were originally proposed in [1, 2] (see [3-28] for some further developments). Flavour physics in these models has been studied in [9, 10, 13, $17-$ 19, 27, 30]. The models describe locally intersecting 7-branes within a Calabi-Yau fourfold by a Higgsed twisted 8-dimensional gauge theory. The Higgsing induces localised zero modes along curves in the four-dimensional manifold $S$ wrapped by the 7-brane. In the presence of flux, these zero modes correspond to chiral matter fields in the four-dimensional effective GUT. The existence of a tree-level top Yukawa coupling requires the gauge theory to have an underlying exceptional gauge group, thereby enforcing the non-perturbative F-theory approach.

Following the initial work an attractive proposal was made in [10] where the hierarchical structure of the Yukawas was proposed to arise from a rank one Yukawa matrix via perturbative corrections in powers of flux. Furthermore the perturbative parameter was argued to be related to the GUT gauge coupling. The resulting CKM matrix, following 
some assumptions regarding order one factors and the loci where up-type and down-type Yukawas where generated, took a phenomenologically attractive form. This proposal was studied in more detail in subsequent papers $[18,24,27]$. These papers, as initial studies of flavour, did not deeply explore the effects and constraints of supersymmetry. The aim of this article is to study the connection between these proposed models of flavour and the constraints arising from supersymmetry.

This paper is organised as follows. In section 2 we review the topologically twisted theory as described in [2]. In section 3 we relate this theory to dimensional reduction of the canonical presentation of $8 \mathrm{~d}$ super Yang-Mills. We show how the the equations of motion and Yukawa coupling take an identical form in the two approaches and give a dictionary between the two formalisms. We show how solutions to the equations of motion are unaltered under overall metric rescalings. This is important since the volume of $S$ gives the gauge coupling of the effective four-dimensional GUT. We relate this to the constraints required by holomorphy of the effective $4 \mathrm{~d}$ supergravity theory and explain why $\alpha_{\text {GUT }}$ must appear universally in the Yukawa couplings.

In section 4 we study more specifically the equations of motion. In particular we study the effect that solving the D-terms has on the form of the localised matter wavefunctions. For general flux a flat metric on $S$ no longer solves the D-term equations. In order to maintain supersymmetry the metric is deformed, modifying the equations of motion and subsequently the matter wavefunctions. We solve for the corrected wavefunctions at leading order in the perturbations. We find that the metric perturbations dominate over the flux in the wavefunctions and give the leading corrections to the fluxless flat-metric case. Finally we use the resulting wavefunctions to calculate the resulting Yukawa couplings. We find that, other than the tree-level top Yukawa, all the Yukawa couplings vanish exactly.

Note added. While this manuscript was in preparation the paper [30] appeared studying similar issues. There the vanishing of the Yukawa couplings was demonstrated and explained at a deeper level than that which appears in this paper. We refer the reader to [30] for a more fundamental explanation for the vanishing of Yukawa couplings.

\section{Local models of intersecting branes in F-theory}

In this section we review the topologically twisted theory of [2] that describes local models of intersecting 7-branes in F-theory. In section 3 we shall relate this theory to the direct dimensional reduction of 8-dimensional Super-Yang-Mills (SYM) and provide a dictionary between the two formulations.

\subsection{Effective theory for intersecting 7-branes}

The relevant theory is an 8-dimensional twisted (off-shell supersymmetric) gauge theory, with gauge group $G$, describing a 7-brane wrapping a 4-dimensional Kähler hypersurface $S$. For a local model $S$ is a shrinkable manifold (more formally it has an ample normal bundle) within a Calabi-Yau (CY) 4-fold $X$. The 8-dimensional fields are given in terms 
of adjoint valued, $S$-valued, 4-dimensional $N=1$ multiplets

$$
\begin{aligned}
\mathbf{A}_{\bar{m}} & =\left(A_{\bar{m}}, \psi_{\bar{m}}, G_{\bar{m}}\right), \\
\mathbf{\Phi}_{m n} & =\left(\varphi_{m n}, \chi_{m n}, H_{m n}\right), \\
\mathbf{V} & =\left(\eta, A_{\mu}, D\right) .
\end{aligned}
$$

The indices on the fields denote their form-values on $S$. So for example $A_{\bar{m}} \in \bar{\Omega}_{S}^{1} \otimes \operatorname{ad}(P)$ where $\Omega_{S}^{p}$ denotes holomorphic $p$-form on $S$ and $P$ is the principle bundle (in the adjoint representation) associated to the gauge group $G$. Here $\mathbf{A}$ and $\mathbf{\Phi}$ are chiral multiplets with respective F-terms $G$ and $H . \mathbf{V}$ is a vector multiplet with D-term $D . A_{\bar{m}}$ and $\varphi_{m n}$ are complex scalars while $\psi_{\bar{m}}, \chi_{m n}$, and $\eta$ are fermions.

The action for the effective theory was given in [2]. Setting 4-dimensional variations of the fields to zero, the equations of motion that follow are

$$
\begin{aligned}
H-F^{(2,0)} & =0, \\
i[\varphi, \bar{\varphi}]+2 \omega \wedge F^{(1,1)}+\star_{S} D & =0, \\
2 i \omega \wedge \bar{G}-\bar{\partial}_{A} \varphi & =0, \\
-\partial \bar{H}+2 \omega \wedge \bar{\partial} D+\bar{G} \wedge \bar{\varphi}-\bar{\chi} \wedge \bar{\psi}-i 2 \sqrt{2} \omega \wedge \eta \wedge \psi & =0, \\
\omega \wedge \partial_{A} \psi+\frac{i}{2}[\bar{\varphi}, \chi] & =0, \\
\bar{\partial}_{A} \chi-2 i \sqrt{2} \omega \wedge \partial_{A} \eta-[\varphi, \psi] & =0, \\
\bar{\partial}_{A} \psi-\sqrt{2}[\bar{\varphi}, \eta] & =0, \\
-\sqrt{2}[\bar{\eta}, \bar{\chi}]-\bar{\partial}_{A} G-\frac{1}{2}[\psi, \psi] & =0 .
\end{aligned}
$$

These are the equations that we work with in this paper and they apply to a general Kähler manifold $S$ of large enough volume to neglect $\alpha^{\prime}$ corrections. Equation (2.4) imposes that the flux must be of type $(1,1)$. Equation $(2.5)$ is the D-term equation that we discuss in detail in section 4.1. In this paper we are concerned with vacua where the vacuum expectation value (vev) of $\varphi$, denoted $\langle\varphi\rangle$, and that of $A_{\bar{m}}$, which is responsible for the flux, take values in the Cartan of $G$. Therefore equation (2.6) just imposes that $\langle\varphi\rangle$ is holomorphic

$$
\bar{\partial}_{A}\langle\varphi\rangle=\bar{\partial}\langle\varphi\rangle+[A,\langle\varphi\rangle]=\bar{\partial}\langle\varphi\rangle=0 .
$$

We are interested in solving the equations on a vacuum with

$$
\langle\chi\rangle=\langle\psi\rangle=\langle\eta\rangle=0,
$$

which means that, using equations (2.4)-(2.6) equations (2.7) and (2.11) are satisfied. Equation (2.10) follows from (2.9) once (2.13) is imposed.

We take the manifold $S$ to be Kähler and spanned by two complex coordinates $z_{1}$ and $z_{2}$. We will also restrict to the metric ansatz

$$
d s^{2}=h_{1}\left(z_{1}, \bar{z}_{\overline{1}}\right) d z_{1} \otimes d \bar{z}_{\overline{1}}+h_{2}\left(z_{2}, \bar{z}_{\overline{2}}\right) d z_{2} \otimes d \bar{z}_{\overline{2}},
$$


where $h_{1}$ and $h_{2}$ are general functions of $z_{1}$ and $z_{2}$ respectively (this is the form of the metric we will use when solving for explicit wavefunctions in section 4). The corresponding Kähler form is given by

$$
\omega=\frac{i}{2} h_{1} d z_{1} \wedge d \bar{z}_{\overline{1}}+\frac{i}{2} h_{2} d z_{2} \wedge d \bar{z}_{\overline{2}} .
$$

Putting this into (2.4)-(2.11) the relevant equations read

$$
\begin{aligned}
h_{1} \partial_{A 2} \psi_{\overline{2}}+h_{2} \partial_{A 1} \psi_{\overline{1}}-[\bar{\varphi}, \chi] & =0, \\
\bar{\partial}_{A \overline{1}} \chi-\left[\varphi, \psi_{\overline{1}}\right] & =0, \\
\bar{\partial}_{A \overline{2}} \chi-\left[\varphi, \psi_{\overline{2}}\right] & =0,
\end{aligned}
$$

where by abuse of notation we have relabeled $\varphi_{12} \rightarrow \varphi$ and $\chi_{12} \rightarrow \chi$. Apart from these equations we also have the D-term equation (2.5).

\subsection{Zero modes and Yukawa couplings}

Consider turning off the flux so that there is no gauge field background. We then turn on a vev for $\varphi$ given by

$$
\langle\varphi\rangle=m_{1}^{2} z_{1} Q_{1}+m_{2}^{2} z_{2} Q_{2} .
$$

Here $Q_{1}$ and $Q_{2}$ are elements in $G$. In this paper we perform the simplification $m_{1}=m_{2}=$ $m$ and define

$$
v \equiv \frac{1}{m^{2}} .
$$

Since there is no flux the D-term equation is solved identically and we are free to consider a flat metric background $h_{1}=h_{2}=1$. The equations of motion to be solved then read

$$
\begin{aligned}
\partial_{2} \psi_{\overline{2}}+\partial_{1} \psi_{\overline{1}}-\frac{1}{v}\left(\bar{z}_{1} q_{1}+\bar{z}_{2} q_{2}\right) \chi & =0, \\
\bar{\partial}_{\overline{1}} \chi-\frac{1}{v}\left(z_{1} q_{1}+z_{2} q_{2}\right) \psi_{\overline{1}} & =0, \\
\bar{\partial}_{\overline{2}} \chi-\frac{1}{v}\left(z_{1} q_{1}+z_{2} q_{2}\right) \psi_{\overline{2}} & =0 .
\end{aligned}
$$

Here $q_{1}$ and $q_{2}$ are the charges of the fields under $Q_{1}$ and $Q_{2}$. For simplicity consider the case $q_{1}=1$ and $q_{2}=0$. Then the equations have a localised solution given by

$$
\chi=f\left(z_{2}\right) e^{-\frac{\left|z_{1}\right|^{2}}{v}} .
$$

Here and in section 4 , when presenting a solution we only present the expression for $\chi$ since the expressions for $\psi_{\overline{1}}$ and $\psi_{\overline{2}}$ can be determined directly from it using (2.22) and (2.23). This is a localised zero mode which can be thought of as strings stretching between two 7 -branes that are intersecting along $z_{1}=0$. If we decompose the 8-dimensional fields into external space-time 4-dimensional components and internal 4-dimensional components along $S$ we see that the equations imply that the external 4-dimensional components of $\chi$, $\psi_{\overline{1}}$ and $\psi_{\overline{2}}$ are all equal and they count as a single 4-dimensional zero mode. 
If flux is turned on there are two changes: the form of the internal wavefunctions changes and the number of zero modes can change. The change in the form of the wavefunctions is a local effect that we calculate explicitly in section 4. The replication of zero modes is of course the family index and depends on the global properties of the flux. In a local model we decouple the connection between the flux and the zero mode replication.

The Yukawa couplings are associated to wavefunction overlaps on a point of intersection of three curves where the gauge symmetry is enhanced. For example the bottom type Yukawas are associated to a point of $S O(12)$ gauge symmetry. The matter arises from the decomposition of the adjoint

$$
\begin{aligned}
S O(12) & \supset S U(5) \times \mathrm{U}(1)_{\mathrm{a}} \times \mathrm{U}(1)_{\mathrm{b}}, \\
\mathbf{6 6} & \rightarrow \mathbf{2 4}^{(\mathbf{0 , 0})} \oplus \mathbf{1}^{(\mathbf{0 , 0}, \mathbf{0})} \oplus \mathbf{1}^{(\mathbf{0}, \mathbf{0})} \oplus(\mathbf{5} \oplus \overline{\mathbf{5}})^{(-\mathbf{1}, \mathbf{0})} \oplus(\mathbf{5} \oplus \overline{\mathbf{5}})^{(\mathbf{1}, \mathbf{1})} \oplus(\mathbf{1 0} \oplus \overline{\mathbf{1 0}})^{(\mathbf{0 , 1})},
\end{aligned}
$$

where the superscripts denote the charges of the $\mathbf{5}$ and $\mathbf{1 0}$. Then we see that the $\overline{\mathbf{5}}_{H} \overline{\mathbf{5}}_{M} \mathbf{1 0}_{M}$ Yukawa, corresponds to curves of charges $(-1,-1),(1,0)$ and $(0,1)$ respectively. We label the matter from each of the three curves with the index $\lambda=1,2,3$ corresponding to $\overline{\mathbf{5}}_{H}$, $\overline{\mathbf{5}}_{M}$ and $\mathbf{1 0}_{M}$ respectively. The fields are therefore the sum over the localised solutions

$$
\chi=\sum_{\lambda} \chi^{\lambda}, \quad \psi=\sum_{\lambda} \psi^{\lambda}
$$

Since the equations of motion are linear they exactly decouple and can be solved separately for each component.

There is also a family index $a=1,2,3$ which counts copies of the zero modes associated to global properties of the flux. A four-dimensional $\phi$ field is then labeled by

$$
\phi^{(\lambda, a)}:\left\{\chi^{(\lambda, a)}, \psi_{\overline{1}}^{(\lambda, a)}, \psi_{\overline{2}}^{(\lambda, a)}\right\}
$$

Since there is only one Higgs generation for $\lambda=1$ we only have $a=1$.

The relevant Yukawa coupling are the sum over all the terms arising from

$$
\int_{S} A \wedge A \wedge \Phi=-\int_{S} \hat{\psi}_{\overline{1}}^{i} \hat{\psi}_{\overline{2}}^{j} \hat{\chi}_{12}^{k} 2 \operatorname{Tr}\left(\left[t_{i}, t_{j}\right] t_{k}\right) d z_{1} \wedge d \bar{z}_{\overline{1}} \wedge d z_{2} \wedge d \bar{z}_{\overline{2}}
$$

Here $t_{i}$ are generators of the point gauge group $S O(12)$. Under the decomposition these correspond to curve indices, $\lambda$ in (2.27), with

$$
\lambda=1: \mathbf{5}_{H}, \quad \lambda=2: \mathbf{5}_{M}, \quad \lambda=3: \mathbf{1 0}_{M} .
$$

We therefore obtain the Yukawas

$$
Y^{a b}=a \int_{S}\left[\hat{\psi}_{\overline{1}}^{1} \hat{\psi}_{\overline{2}}^{3, b} \hat{\chi}^{2, a}+\hat{\psi}_{\overline{1}}^{2, a} \hat{\psi}_{\overline{2}}^{1} \hat{\chi}^{3, b}-\hat{\psi}_{\overline{1}}^{2, a} \hat{\psi}_{\overline{2}}^{3, b} \hat{\chi}^{1}-\hat{\psi}_{\overline{1}}^{1} \hat{\psi}_{\overline{2}}^{2, a} \hat{\chi}^{3, b}-\hat{\psi}_{\overline{1}}^{3, b} \hat{\psi}_{\overline{2}}^{1} \hat{\chi}^{2, a}+\hat{\psi}_{\overline{1}}^{3, b} \hat{\psi}_{\overline{2}}^{2, a} \hat{\chi}^{1}\right] \epsilon .
$$

Here $\epsilon$ is the canonical volume form as in (2.28). The constant $a$ is a normalisation factor which is used to normalise the top Yukawa to 1. 


\section{$3 \quad$ Relationship to super Yang-Mills}

Above we have reviewed, following [2], the topological equations for the dynamics of the 8-dimensional field theory. In this section we show how these equations arise from direct analysis of the canonical 8d SYM Lagrangian and provide a dictionary that translates between the fields of the canonical presentation of 8d SYM and the fields of the topologically twisted theory used in [2]. While ultimately the equations are identical, this provides an alternative perspective on the topological theory of [2] and may make certain properties of the theory more intuitive.

$8 \mathrm{~d}$ super Yang-Mills is most easily obtained by dimensional reduction of the $10 \mathrm{~d}$ theory. The $10 \mathrm{~d}$ action is

$$
\int \sqrt{g} d^{10} x\left(-\frac{1}{4} \operatorname{Tr}\left(F_{M N} F^{M N}\right)-\frac{1}{2} \operatorname{Tr}\left(\bar{\lambda} \Gamma^{M} \mathcal{D}_{M} \lambda\right)\right),
$$

where $\lambda$ is a Majorana-Weyl spinor and $\mathcal{D}_{M} X=\partial_{m} X-i\left[A_{m}, X\right]$. Dimensional reduction gives the 8-dimensional action,

$$
\int d^{8} x \sqrt{g}\left(-\frac{1}{4} \operatorname{Tr}\left(F_{M N} F^{M N}\right)-\frac{1}{4} \operatorname{Tr}\left(\mathcal{D}_{M} \phi \mathcal{D}^{M} \phi^{*}\right)+\text { quartics }-\frac{1}{2} \operatorname{Tr}\left(\bar{\lambda} \Gamma^{m} D_{m} \lambda\right)+\frac{i}{2} \bar{\lambda} \Gamma^{r}\left[\phi_{r}, \lambda\right]\right)
$$

We drop quartic scalar interactions as they are not relevant for our purposes.

Let us now describe $\lambda$. It is convenient to view $\lambda$ as a 10-dimensional Majorana-Weyl spinor with 8 complex degrees of freedom (before imposing equations of motion). We follow [29] to write the flat space gamma matrices as $(\mu=0,1,2,3, m=4,5,6,7, r=8,9)$

$$
\Gamma^{\mu}=\gamma^{\mu} \otimes \mathbb{I} \otimes \mathbb{I}, \quad \Gamma^{m}=\gamma^{5} \otimes \tilde{\gamma}^{m} \otimes \mathbb{I}, \quad \Gamma^{r}=\gamma^{5} \otimes \tilde{\gamma}^{5} \otimes \tau^{r},
$$

with

$$
\begin{aligned}
& \gamma^{0}=\left(\begin{array}{c}
0-\mathbb{I} \\
\mathbb{I} 0
\end{array}\right), \quad \gamma^{1}=\left(\begin{array}{cc}
0 & \sigma_{x} \\
\sigma_{x} & 0
\end{array}\right), \quad \gamma^{2}=\left(\begin{array}{cc}
0 & \sigma_{y} \\
\sigma_{y} & 0
\end{array}\right), \quad \gamma^{3}=\left(\begin{array}{cc}
0 & \sigma_{z} \\
\sigma_{z} & 0
\end{array}\right), \quad \gamma^{5}=\left(\begin{array}{cc}
1 & 0 \\
0 & -1
\end{array}\right),
\end{aligned}
$$

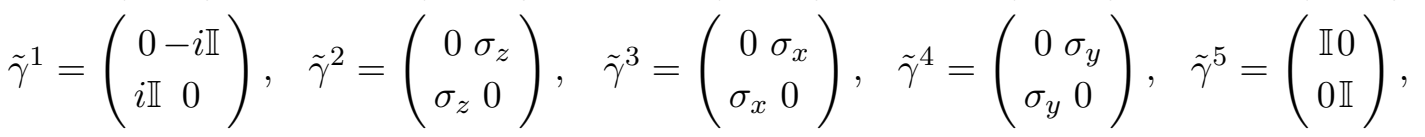

and

$$
\tau^{8}=\sigma_{x}=\left(\begin{array}{ll}
0 & 1 \\
1 & 0
\end{array}\right), \quad \tau^{9}=\sigma_{y}=\left(\begin{array}{cc}
0 & -i \\
i & 0
\end{array}\right) .
$$

Using $u$ to denote the complexified 8,9 directions, we also note

$$
\tau^{u} \equiv \sigma_{x}+i \sigma_{y}=2\left(\begin{array}{ll}
0 & 1 \\
0 & 0
\end{array}\right), \quad \tau^{\bar{u}} \equiv \sigma_{x}-i \sigma_{y}=2\left(\begin{array}{ll}
0 & 0 \\
1 & 0
\end{array}\right),
$$

Spinors can be decomposed by their chiralities under each of the three $(4 d+4 d+2 d)$ components of the gamma matrices. The Majorana condition can be written as $\lambda^{*}=B \lambda$, where $B=\Gamma^{2} \Gamma^{4} \Gamma^{7} \Gamma^{9}$. 
As in [29] it can be shown that a general Majorana-Weyl spinor can be written as

$$
\lambda=\left(\lambda_{1}+\lambda_{4}\right) \oplus\left(\lambda_{2}+\lambda_{3}\right)
$$

where $\left(\lambda_{1}, \lambda_{2}, \lambda_{3}, \lambda_{4}\right)=\left\{\lambda^{a b \alpha}, \lambda_{b \alpha}^{a}, \lambda_{a b}{ }^{\alpha}, \lambda_{a \alpha}{ }^{b}\right)$. Super/sub scripts indicate positive or negative chirality in that direction. The notation $\left(\lambda_{1}+\lambda_{4}\right)$ indicates that the components of $\lambda_{1}$ and $\lambda_{4}$ are not independent: they are related by the Majorana condition. More precisely, we have

$$
\begin{aligned}
& \lambda_{1}+\lambda_{4}=\left(\begin{array}{c}
\xi_{1} \\
0
\end{array}\right) \otimes\left(\begin{array}{c}
\psi_{1} \\
0
\end{array}\right) \otimes\left(\begin{array}{c}
\theta_{1} \\
0
\end{array}\right)+\left(\begin{array}{c}
0 \\
-\sigma_{y} \xi_{1}^{*}
\end{array}\right) \otimes\left(\begin{array}{c}
-i \sigma_{y} \psi_{1}^{*} \\
0
\end{array}\right) \otimes\left(\begin{array}{c}
0 \\
-i \theta_{1}^{*}
\end{array}\right), \\
& \lambda_{2}+\lambda_{3}=\left(\begin{array}{c}
\xi_{2} \\
0
\end{array}\right) \otimes\left(\begin{array}{c}
0 \\
\psi_{2}
\end{array}\right) \otimes\left(\begin{array}{c}
0 \\
\theta_{2}
\end{array}\right)+\left(\begin{array}{c}
0 \\
-\sigma_{y} \xi_{2}^{*}
\end{array}\right) \otimes\left(\begin{array}{c}
0 \\
-i \sigma_{y} \psi_{2}^{*}
\end{array}\right) \otimes\left(\begin{array}{c}
i \theta_{2}^{*} \\
0
\end{array}\right) .
\end{aligned}
$$

$\lambda_{4}$ and $\lambda_{3}$ correspond to the CPT conjugates of $\lambda_{1}$ and $\lambda_{2}$ respectively, and thus do not represent physically distinct degrees of freedom.

For the extra-dimensional spinor components we will also often write

$$
\left(\begin{array}{c}
\psi_{1} \\
\psi_{2}
\end{array}\right) \rightarrow\left(\begin{array}{l}
\psi_{1,1} \\
\psi_{1,2} \\
\psi_{2,1} \\
\psi_{2,2}
\end{array}\right)
$$

\subsection{Equations of motion}

We need to account for the non-trivial metric on the surface wrapped by the branes. For this we suppose we start with a metric of the factorised form

$$
d s^{2}=4 h_{1}(z, \bar{z}) d z d \bar{z}+4 h_{2}(w, \bar{w}) d w d \bar{w},
$$

so $g_{z \bar{z}}=2 h_{1}(z, \bar{z}), g_{w \bar{w}}=2 h_{2}(w, \bar{w}), g^{z \bar{z}}=\frac{1}{2 h_{1}(z, \bar{z})}, g^{w \bar{w}}=\frac{1}{2 h_{2}(w, \bar{w})}$. The spin connection is given by

$$
\omega_{\mu}^{a b}=\frac{1}{2} e^{a \nu}\left(\partial_{\mu} e_{\nu}^{b}-\partial_{\nu} e_{\mu}^{b}\right)-\frac{1}{2} e^{b \nu}\left(\partial_{\mu} e_{\nu}^{a}-\partial_{\nu} e_{\mu}^{a}\right)-\frac{1}{2} e^{\psi a} e^{\sigma b}\left(\partial_{\psi} e_{\sigma c}-\partial_{\sigma} e_{\psi c}\right) e_{\mu}^{c}
$$

Here $a, b$ are the vielbein indices with a flat metric while $\mu, \nu$ are the spacetime indices that run over $z, \bar{z}, w, \bar{w}$. As a vielbein we take

$$
\begin{array}{llll}
e_{z}^{1}=h_{1}^{\frac{1}{2}}, & e_{\bar{z}}^{1}=h_{1}^{\frac{1}{2}}, & e_{w}^{1}=0, & e_{\bar{w}}^{1}=0 . \\
e_{z}^{2}=i h_{1}^{\frac{1}{2}}, & e_{\bar{z}}^{2}=-i h_{1}^{\frac{1}{2}}, & e_{w}^{2}=0, & e_{\bar{w}}^{2}=0 . \\
e_{z}^{3}=0, & e_{\bar{z}}^{3}=0, & e_{w}^{3}=h_{2}^{\frac{1}{2}}, & e_{\bar{w}}^{3}=h_{2}^{\frac{1}{2}} . \\
e_{z}^{4}=0, & e_{\bar{z}}^{4}=0, & e_{w}^{4}=i h_{2}^{\frac{1}{2}}, & e_{\bar{w}}^{4}=-i h_{2}^{\frac{1}{2}} .
\end{array}
$$

From these we can compute the non-vanishing elements of the spin connection to be

$$
\omega_{z}^{12}=-\frac{i}{2 h_{1}} \partial_{z} h_{1}, \quad \omega_{\bar{z}}^{12}=\frac{i}{2 h_{1}} \partial_{\bar{z}} h_{1}, \quad \omega_{w}^{34}=-\frac{i}{2 h_{2}} \partial_{w} h_{2}, \quad \omega_{\bar{w}}^{34}=\frac{i}{2 h_{2}} \partial_{\bar{w}} h_{2} .
$$


We also need to determine the gamma matrices. We have

$$
\begin{array}{ll}
\tilde{\gamma}^{z}=e^{z 1} \tilde{\gamma}_{1}+e^{z 2} \tilde{\gamma}_{2}=\frac{h_{1}^{-1 / 2}}{2}\left(\tilde{\gamma}_{1}-i \tilde{\gamma}_{2}\right), & \tilde{\gamma}^{\bar{z}}=e^{\bar{z} 1} \tilde{\gamma}_{1}+e^{\bar{z} 2} \tilde{\gamma}_{2}=\frac{h_{1}^{-1 / 2}}{2}\left(\tilde{\gamma}_{1}+i \tilde{\gamma}_{2}\right), \\
\tilde{\gamma}^{w}=\frac{h_{2}^{-\frac{1}{2}}}{2}\left(\tilde{\gamma}_{3}-i \tilde{\gamma}_{4}\right), & \tilde{\gamma}^{\bar{w}}=\frac{h_{2}^{-\frac{1}{2}}}{2}\left(\tilde{\gamma}_{3}+i \tilde{\gamma}_{4}\right) .
\end{array}
$$

Therefore we can write

$$
\begin{aligned}
& \tilde{\gamma}^{z}=\frac{h_{1}^{-\frac{1}{2}}}{2}\left(\begin{array}{cccc}
0 & 0 & -2 i & 0 \\
0 & 0 & 0 & 0 \\
0 & 0 & 0 & 0 \\
0 & 2 i & 0 & 0
\end{array}\right), \quad \tilde{\gamma}^{\bar{z}}=\frac{h_{1}^{-\frac{1}{2}}}{2}\left(\begin{array}{cccc}
0 & 0 & 0 & 0 \\
0 & 0 & 0 & -2 i \\
2 i & 0 & 0 & 0 \\
0 & 0 & 0 & 0
\end{array}\right), \\
& \tilde{\gamma}^{w}=\frac{h_{2}^{-\frac{1}{2}}}{2}\left(\begin{array}{llll}
0 & 0 & 0 & 0 \\
0 & 0 & 2 & 0 \\
0 & 0 & 0 & 0 \\
2 & 0 & 0 & 0
\end{array}\right), \quad \tilde{\gamma}^{\bar{w}}=\frac{h_{2}^{-\frac{1}{2}}}{2}=\left(\begin{array}{llll}
0 & 0 & 0 & 2 \\
0 & 0 & 0 & 0 \\
0 & 2 & 0 & 0 \\
0 & 0 & 0 & 0
\end{array}\right) \text {. }
\end{aligned}
$$

For the spin connection we need $\frac{1}{8} \gamma^{M} \omega_{M, \alpha \beta}\left[\gamma^{\alpha}, \gamma^{\beta}\right]$. As $\left[\gamma^{1}, \gamma^{2}\right]=-2 i\left(\begin{array}{cc}\sigma_{z} & 0 \\ 0 & -\sigma_{z}\end{array}\right)$,

$$
\begin{aligned}
& {\left[\gamma^{3}, \gamma^{4}\right]=2 i\left(\begin{array}{cc}
\sigma_{z} & 0 \\
0 & \sigma_{z}
\end{array}\right) \text {, we have }} \\
& \frac{1}{8} \omega_{z \alpha \beta}\left[\gamma^{\alpha}, \gamma^{\beta}\right]=-\frac{\partial_{z} h_{1}}{4 h_{1}}\left(\begin{array}{cc}
\sigma_{z} & 0 \\
0 & -\sigma_{z}
\end{array}\right), \quad \frac{1}{8} \omega_{\bar{z} \alpha \beta}\left[\gamma^{\alpha}, \gamma^{\beta}\right]=\frac{\partial_{\bar{z}} h_{1}}{4 h_{1}}\left(\begin{array}{cc}
\sigma_{z} & 0 \\
0 & -\sigma_{z}
\end{array}\right) . \\
& \frac{1}{8} \omega_{w \alpha \beta}\left[\gamma^{\alpha}, \gamma^{\beta}\right]=\frac{\partial_{w} h_{2}}{4 h_{2}}\left(\begin{array}{cc}
\sigma_{z} & 0 \\
0 & \sigma_{z}
\end{array}\right), \quad \frac{1}{8} \omega_{\bar{w} \alpha \beta}\left[\gamma^{\alpha}, \gamma^{\beta}\right]=\frac{\partial_{\bar{w}} h_{2}}{4 h_{2}}\left(\begin{array}{cc}
\sigma_{z} & 0 \\
0 & \sigma_{z}
\end{array}\right) \text {. }
\end{aligned}
$$

Putting these together we obtain

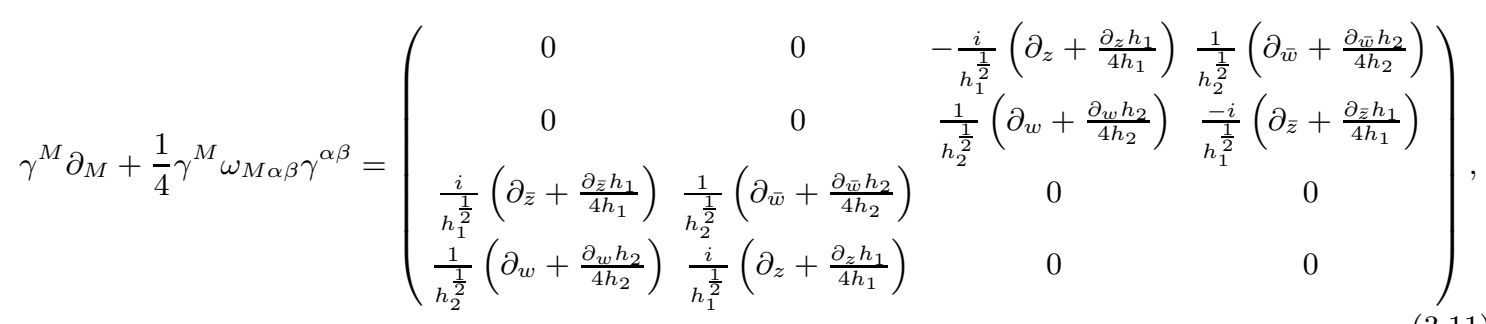

where $\gamma^{\alpha \beta} \equiv \frac{1}{2}\left[\gamma^{\alpha}, \gamma^{\beta}\right]$. We need to twist this operator to account for the nontrivial metric. The twisting corresponds to an additional gauge field in the canonical bundle. To determine this, let us first recall that a metric

$$
d s^{2}=4 h_{1}(z, \bar{z}) d z d \bar{z}+4 h_{2}(w, \bar{w}) d w d \bar{w}
$$

has

$$
\Gamma_{z z}^{z}=\frac{\partial_{z} h_{1}}{h_{1}}, \quad \Gamma_{\bar{z} \bar{z}}^{\bar{z}}=\frac{\partial_{\bar{z}} h_{1}}{h_{1}}, \quad \Gamma_{w w}^{w}=\frac{\partial_{w} h_{2}}{h_{2}}, \quad \Gamma_{\bar{w} \bar{w}}^{\bar{w}}=\frac{\partial_{\bar{w}} h_{2}}{h_{2}}
$$


The twisting corresponds to a gauge field in the canonical bundle. A gauge field with $M$ units of flux in the $z \bar{z}$ direction and $N$ units of flux in the $w \bar{w}$ direction has

$$
A_{z}=-\frac{i M \partial_{z} h_{1}}{4 h_{1}}, \quad A_{\bar{z}}=\frac{i M \partial_{\bar{z}} h_{1}}{4 h_{1}}, \quad A_{w}=-\frac{i N \partial_{w} h_{2}}{4 h_{2}}, \quad A_{\bar{w}}=\frac{i N \partial_{\bar{w}} h_{2}}{4 h_{2}}
$$

The factor of $i / 4$ can be determined by reference to the case of $\mathbb{P}^{1} \times \mathbb{P}^{1}$, for which $z$ and $w$ parameterise the two separate $\mathbb{P}^{1} \mathrm{~s}$. This gives

$$
\begin{aligned}
& \gamma^{M} \partial_{M}+\frac{1}{4} \gamma^{M} \omega_{M \alpha \beta} \gamma^{\alpha \beta}-i \gamma^{M} A_{M}= \\
& \left(\begin{array}{cccc}
0 & 0 & \frac{-i}{h_{1}^{\frac{1}{2}}}\left(\partial_{z}+\frac{\partial_{z} h_{1}}{4 h_{1}}(1-M)\right) & \frac{1}{h_{2}^{\frac{1}{2}}}\left(\partial_{\bar{w}}+\frac{\partial_{\bar{w}} h_{2}}{4 h_{2}}(1+N)\right) \\
0 & 0 & \frac{1}{h_{2}^{\frac{1}{2}}}\left(\partial_{w}+\frac{\partial_{w} h_{2}}{4 h_{2}}(1-N)\right) & \frac{-i}{h_{1}^{\frac{1}{2}}}\left(\partial_{\bar{z}}+\frac{\partial_{\bar{z}} h_{1}}{4 h_{1}}(1+M)\right) \\
\frac{i}{h_{1}^{\frac{1}{2}}}\left(\partial_{\bar{z}}+\frac{\partial_{z} h_{1}}{4 h_{1}}(1+M)\right) & \frac{1}{h_{2}^{\frac{1}{2}}}\left(\partial_{\bar{w}}+\frac{\partial_{\bar{w}} h_{2}}{4 h_{2}}(1+N)\right) & 0 & 0 \\
\frac{1}{h_{2}^{\frac{1}{2}}}\left(\partial_{w}+\frac{\partial_{w} h_{2}}{4 h_{2}}(1-N)\right) \frac{i}{h_{1}^{\frac{1}{2}}}\left(\partial_{z}+\frac{\partial_{z} h_{1}}{4 h_{1}}(1-M)\right) & 0 & 0
\end{array}\right)
\end{aligned}
$$

As discussed in [2, 29], the left- and right-handed spinors $\psi_{1}$ and $\psi_{2}$ have opposite Rcharges and so should be twisted in opposite directions. To accomplish this we modify the Dirac operator (3.13) by

$$
\begin{array}{ll}
(M, N) \rightarrow(M, N)+(1,1) & \text { for }\left(\psi_{1,1}, \psi_{1,2}\right), \\
(M, N) \rightarrow(M, N)-(1,1) & \text { for }\left(\psi_{2,1}, \psi_{2,2}\right) .
\end{array}
$$

The twisted Dirac operator is then

$$
\begin{aligned}
& \gamma^{M} \partial_{M}+\frac{1}{4} \gamma^{M} \omega_{M \alpha \beta} \gamma^{\alpha \beta}-i \gamma^{M} A_{\text {twisted }, M}= \\
& \left(\begin{array}{cccc}
0 & 0 & \frac{-i}{h_{1}^{\frac{1}{2}}}\left(\partial_{z}+\frac{\partial_{z} h_{1}}{4 h_{1}}(2-M)\right) & \frac{1}{h_{2}^{\frac{1}{2}}}\left(\partial_{\bar{w}}+N \frac{\partial_{\bar{w}} h_{2}}{4 h_{2}}\right) \\
0 & 0 & \frac{1}{h_{2}^{\frac{1}{2}}}\left(\partial_{w}+\frac{\partial_{w} h_{2}}{4 h_{2}}(2-N)\right) & \frac{-i}{h_{1}^{\frac{1}{2}}}\left(\partial_{\bar{z}}+M \frac{\partial_{\bar{z}} h_{1}}{4 h_{1}}\right) \\
\frac{i}{h_{1}^{\frac{1}{2}}}\left(\partial_{\bar{z}}+\frac{\partial_{\bar{z}} h_{1}}{4 h_{1}}(2+M)\right) & \frac{1}{h_{2}^{\frac{1}{2}}}\left(\partial_{\bar{w}}+\frac{\partial_{\bar{w}} h_{2}}{4 h_{2}}(2+N)\right) & 0 & 0 \\
\frac{1}{h_{2}^{\frac{1}{2}}}\left(\partial_{w}-N \frac{\partial_{w} h_{2}}{4 h_{2}}\right) & \frac{i}{h_{1}^{\frac{1}{2}}}\left(\partial_{z}-M \frac{z_{z} h_{1}}{4 h_{1}}\right) & 0 & 0
\end{array}\right) .
\end{aligned}
$$

For compactness we write

$$
\gamma^{M} \partial_{M}+\frac{1}{4} \gamma^{M} \omega_{M \alpha \beta} \gamma^{\alpha \beta}-i \gamma^{M} A_{t w i s t e d, M} \equiv \gamma^{M} \mathcal{D}_{M} \equiv\left(\begin{array}{cc}
0 & \mathcal{D}_{-} \\
\mathcal{D}_{+} & 0
\end{array}\right)
$$

Note that for $M=N=0, \psi_{2,2}=1$ is a solution to $\gamma^{M} \mathcal{D}_{M} \psi=0$. This corresponds to the constant gaugino zero mode which should exist for arbitrarily curved spaces. The general solutions are

$$
\left(\begin{array}{c}
\psi_{1,1} \\
\psi_{1,2} \\
\psi_{2,1} \\
\psi_{2,2}
\end{array}\right)=\left(\begin{array}{c}
h_{1}^{-\frac{M+2}{4}} h_{2}^{\frac{N}{4}} \psi(z, \bar{w}) \\
h_{1}^{\frac{M}{4}} h_{2}^{-\frac{N+2}{4}} \psi(\bar{z}, w) \\
h_{1}^{\frac{M-2}{4}} h_{2}^{\frac{N-2}{4}} \psi(\bar{z}, \bar{w}) \\
h_{1}^{-\frac{M}{4}} h_{2}^{-\frac{N}{4}} \psi(z, w)
\end{array}\right)
$$


To study, at least locally, intersecting branes we also need the profile for the Higgs field, namely the complex scalar $\phi$ that is present in the 8d SYM theory. In an untwisted background this field would satisfy

$$
\partial_{\bar{z}} \phi_{u}=0, \quad \partial_{z} \phi_{\bar{u}}=0, \quad \partial_{\bar{w}} \phi_{u}=0, \quad \partial_{w} \phi_{\bar{u}}=0 .
$$

Zero mode solutions are therefore given by

$$
\mathcal{D}_{z} \phi_{\bar{u}}=\left(\partial_{z}-\frac{\partial_{z} h_{1}}{4 h_{1}} M\right) \phi_{\bar{u}}=0
$$

and so $\phi_{\bar{u}}=h_{1}^{M / 4} \phi(\bar{z})$. Including also the $w$ direction we obtain

$$
\phi_{\bar{u}}=h_{1}^{M / 4} h_{2}^{N / 4} \phi(\bar{z}, \bar{w}),
$$

where $\phi(\bar{z}, \bar{w})$ is antiholomorphic. This field has $R$-charge 2 in contrast to the fermions which had $R$-charge 1 . So when we twist the theory we need to modify the equations of motion by two units of flux, $(M, N) \rightarrow(M-2, N-2)$, rather than the single unit of flux that applied for the fermions. The fact that $M \rightarrow M-2$ rather than $M \rightarrow M+2$ is by analogy with the twist for $\psi_{3}$ which is the fermionic partner of $\phi$.

The Higgs field whose vev separates different brane stacks is only charged due to the twisting (as it is valued in the Cartan it is uncharged under any gauge flux that is turned on). So in fact the twisting gives the sole 'gauge' contribution to the wavefunction, and we have

$$
\begin{aligned}
& \phi_{\bar{u}}=h_{1}^{-1 / 2} h_{2}^{-1 / 2} \phi(\bar{z}, \bar{w}), \\
& \phi_{u}=h_{1}^{-1 / 2} h_{2}^{-1 / 2} \phi(z, w) .
\end{aligned}
$$

In order to use this vev to determine the twisted fermionic equations of motion we need to compute $-i \Gamma^{r}\left[\phi_{r}, \lambda\right]$. Now,

$$
\Gamma^{u}=2\left(\begin{array}{cc}
\mathbb{I} & 0 \\
0 & -\mathbb{I}
\end{array}\right) \otimes\left(\begin{array}{cc}
\mathbb{I} & 0 \\
0 & -\mathbb{I}
\end{array}\right) \otimes\left(\begin{array}{ll}
0 & 1 \\
0 & 0
\end{array}\right), \quad \Gamma^{\bar{u}}=2\left(\begin{array}{cc}
\mathbb{I} & 0 \\
0 & -\mathbb{I}
\end{array}\right) \otimes\left(\begin{array}{cc}
\mathbb{I} & 0 \\
0 & -\mathbb{I}
\end{array}\right) \otimes\left(\begin{array}{ll}
0 & 0 \\
1 & 0
\end{array}\right) .
$$

With

$$
\lambda_{1}=\left(\begin{array}{c}
\psi_{1} \\
0
\end{array}\right) \otimes\left(\begin{array}{l}
1 \\
0
\end{array}\right), \quad \lambda_{2}=\left(\begin{array}{c}
0 \\
\psi_{2}
\end{array}\right) \otimes\left(\begin{array}{l}
0 \\
1
\end{array}\right)
$$

we therefore obtain

$$
i\left(\Gamma^{u} \phi_{u}+\Gamma^{\bar{u}} \phi_{\bar{u}}\right)\left(\lambda_{1}+\lambda_{2}\right)=2 i \phi_{u}\left(\begin{array}{c}
0 \\
\psi_{2}
\end{array}\right) \otimes\left(\begin{array}{l}
1 \\
0
\end{array}\right)-2 i \phi_{\bar{u}}\left(\begin{array}{c}
\psi_{1} \\
0
\end{array}\right) \otimes\left(\begin{array}{l}
0 \\
1
\end{array}\right) .
$$

The equations of motion $\gamma^{M} D_{M} \lambda-i \Gamma^{r}\left[\phi_{r}, \lambda\right]=0$ can also be written using (3.15) as

$$
\begin{aligned}
& \mathcal{D}_{+} \psi_{1}+2 i \phi_{u} \psi_{2}=0 \\
& \mathcal{D}_{-} \psi_{2}-2 i \phi_{\bar{u}} \psi_{1}=0
\end{aligned}
$$


Writing out (3.20) and (3.21) gives

$$
\begin{aligned}
& \left(\begin{array}{cc}
\frac{i}{h_{1}^{\frac{1}{2}}}\left(\partial_{\bar{z}}+\frac{\partial_{\bar{z}} h_{1}}{4 h_{1}}(2+M)\right) & \frac{1}{h_{2}^{\frac{1}{2}}}\left(\partial_{\bar{w}}+\frac{\partial_{\bar{w}} h_{2}}{4 h_{2}}(2+N)\right) \\
\frac{1}{h_{2}^{\frac{1}{2}}}\left(\partial_{w}-N \frac{\partial_{w} h_{2}}{4 h_{2}}\right) & \frac{i}{h_{1}^{\frac{1}{2}}}\left(\partial_{z}-M \frac{\partial_{z} h_{1}}{4 h_{1}}\right)
\end{array}\right)\left(\begin{array}{c}
\psi_{1,1} \\
\psi_{1,2}
\end{array}\right)=-2 i \phi_{u}\left(\begin{array}{l}
\psi_{2,1} \\
\psi_{2,2}
\end{array}\right) . \\
& \left(\begin{array}{cc}
\frac{-i}{\frac{1}{2}}\left(\partial_{z}+\frac{\partial_{z} h_{1}}{4 h_{1}}(2-M)\right) & \frac{1}{\frac{1}{2}}\left(\partial_{\bar{w}}+N \frac{\partial_{\bar{w}} h_{2}}{4 h_{2}}\right) \\
h_{2}^{\frac{1}{2}} \\
\frac{1}{h_{2}^{\frac{1}{2}}}\left(\partial_{w}+\frac{\partial_{w} h_{2}}{4 h_{2}}(2-N)\right) \frac{-i}{\frac{1}{2}}\left(\partial_{\bar{z}}+M \frac{\partial_{\bar{z}} h_{1}}{4 h_{1}}\right)
\end{array}\right)\left(\begin{array}{c}
\psi_{2,1} \\
\psi_{2,2}
\end{array}\right)=+2 i \phi_{\bar{u}}\left(\begin{array}{c}
\psi_{1,1} \\
\psi_{1,2}
\end{array}\right) .
\end{aligned}
$$

As we do not want to excite the gaugino we put $\psi_{2,2}=0$. The second set of equations (3.23) then gives

$$
\begin{aligned}
& \frac{-i}{h_{1}^{\frac{1}{2}}}\left(\partial_{z}+\frac{\partial_{z} h_{1}}{4 h_{1}}(2-M)\right) \psi_{2,1}=2 i \phi_{\bar{u}} \psi_{1,1} \\
& \frac{1}{h_{2}^{\frac{1}{2}}}\left(\partial_{w}+\frac{\partial_{w} h_{2}}{4 h_{2}}(2-N)\right) \psi_{2,1}=2 i \phi_{\bar{u}} \psi_{1,2} .
\end{aligned}
$$

Let us rewrite these equations as

$$
\begin{gathered}
\mathcal{D}_{z}\left(h_{1}^{\frac{1}{2}} h_{2}^{\frac{1}{2}} \psi_{2,1}\right)=\left(\partial_{z}-\frac{M}{4 h_{1}} \partial_{z} h_{1}\right)\left(h_{1}^{\frac{1}{2}} h_{2}^{\frac{1}{2}} \psi_{2,1}\right)=2 \phi_{\bar{u}} h_{1}^{\frac{1}{2}} h_{2}^{\frac{1}{2}}\left(-h_{1}^{\frac{1}{2}} \psi_{1,1}\right), \\
\mathcal{D}_{w}\left(h_{1}^{\frac{1}{2}} h_{2}^{\frac{1}{2}} \psi_{2,1}\right)=\left(\partial_{w}-\frac{N}{4 h_{2}} \partial_{w} h_{2}\right)\left(h_{1}^{\frac{1}{2}} h_{2}^{\frac{1}{2}} \psi_{2,1}\right)=2 \phi_{\bar{u}} h_{1}^{\frac{1}{2}} h_{2}^{\frac{1}{2}}\left(i h_{2}^{\frac{1}{2}} \psi_{1,2}\right) .
\end{gathered}
$$

Using $\psi_{2,2}=0$ in (3.22), and also (3.25) and (3.26), we obtain

$$
\left[\mathcal{D}_{w}\left(\frac{1}{\phi_{\bar{u}} h_{1}^{\frac{1}{2}} h_{2}^{\frac{1}{2}}} \mathcal{D}_{z}\right)-\mathcal{D}_{z}\left(\frac{1}{\phi_{\bar{u}} h_{1}^{\frac{1}{2}} h_{2}^{\frac{1}{2}}} \mathcal{D}_{w}\right)\right]\left(h_{1}^{\frac{1}{2}} h_{2}^{\frac{1}{2}} \psi_{2,1}\right)=0 .
$$

Note (3.27) is automatically satisfied provided $\phi_{\bar{u}} h_{1}^{\frac{1}{2}} h_{2}^{\frac{1}{2}}$ is an anti-holomorphic function of $z$ and $w$, as indeed occurs using (3.18). The commutator $\left[D_{z}, D_{w}\right]$ gives a factor of flux $F_{z w}$ which vanishes for the factorised metric form used.

The upper equation of (3.22) likewise gives

$$
h_{2} \mathcal{D}_{\bar{z}}\left(-h_{1}^{\frac{1}{2}} \psi_{1,1}\right)+h_{1} \mathcal{D}_{\bar{w}}\left(i h_{2}^{\frac{1}{2}} \psi_{1,2}\right)=2\left(h_{1}^{\frac{1}{2}} h_{2}^{\frac{1}{2}} \phi_{u}\right)\left(h_{1}^{\frac{1}{2}} h_{2}^{\frac{1}{2}} \psi_{2,1}\right)
$$

Putting $\phi_{\bar{u}}=h_{1}^{-\frac{1}{2}} h_{2}^{-\frac{1}{2}} \frac{\bar{\phi}(\bar{z}, \bar{w})}{2}$ as in (3.18) (and introducing a factor of 2 for convenience), equations (3.28) (3.25), (3.26) and (3.27) become

$$
\begin{aligned}
h_{2} \mathcal{D}_{\bar{z}}\left(-h_{1}^{\frac{1}{2}} \psi_{1,1}\right)+h_{1} \mathcal{D}_{\bar{w}}\left(i h_{2}^{\frac{1}{2}} \psi_{1,2}\right) & =\phi(z, w)\left(h_{1}^{\frac{1}{2}} h_{2}^{\frac{1}{2}} \psi_{2,1}\right) . \\
\mathcal{D}_{z}\left(h_{1}^{\frac{1}{2}} h_{2}^{\frac{1}{2}} \psi_{2,1}\right) & =\bar{\phi}(\bar{z}, \bar{w})\left(-h_{1}^{\frac{1}{2}} \psi_{1,1}\right) \\
\mathcal{D}_{w}\left(h_{1}^{\frac{1}{2}} h_{2}^{\frac{1}{2}} \psi_{2,1}\right) & =\bar{\phi}(\bar{z}, \bar{w})\left(i h_{2}^{\frac{1}{2}} \psi_{1,2}\right) \\
\frac{1}{\bar{\phi}(\bar{z}, \bar{w})}\left(\mathcal{D}_{w} \mathcal{D}_{z}-\mathcal{D}_{z} \mathcal{D}_{w}\right)\left(h_{1}^{\frac{1}{2}} h_{2}^{\frac{1}{2}} \psi_{2,1}\right) & =0
\end{aligned}
$$


Equations (3.29) to (3.32) can be identified with those coming from the topological theory of [2]. In fact, these equations are precisely identical (up to a complex conjugation which comes from choice of conventions) with equations (2.16) to (2.18). The dictionary to map between the two formulations, namely direct reduction of Super Yang-Mills and the topologically twisted theory, is given by

$$
\begin{aligned}
& -\left.\left.h_{1}^{\frac{1}{2}} \psi_{1,1}\right|_{\mathrm{SYM}} \rightarrow \psi_{z}\right|_{\text {twist }},\left.\left.\quad i h_{2}^{\frac{1}{2}} \psi_{1,2}\right|_{\mathrm{SYM}} \rightarrow \psi_{w}\right|_{\text {twist }},\left.\left.\quad h_{1}^{\frac{1}{2}} h_{2}^{\frac{1}{2}} \psi_{2,1}\right|_{\mathrm{SYM}} \rightarrow \chi^{*}\right|_{\text {twist }}, \\
& \left.\left.h_{1}^{\frac{1}{2}} h_{2}^{\frac{1}{2}} \phi\right|_{\mathrm{SYM}} \rightarrow \phi\right|_{\text {twist }},\left.\left.\quad A\right|_{\mathrm{SYM}} \rightarrow i A\right|_{\text {twist }},\left.\left.\quad \quad \psi_{2,2}\right|_{\mathrm{SYM}} \rightarrow A_{\mu}\right|_{\text {twist }} .
\end{aligned}
$$

We can verify these identifications by applying them to the normalisation of the kinetic terms. The normalisation prefactor for the kinetic terms is given in the 8d SYM theory by

$$
\int_{\Sigma} d^{4} y \sqrt{g} \psi^{\dagger} \psi
$$

applying to all fermions (we shall tend to use $y$ for internal integrals over $S$ and $x$ for integrals over $8 \mathrm{~d}$ space or $4 \mathrm{~d}$ non-compact space). Using the above dictionary it is easy to see that the kinetic terms are

$$
\begin{aligned}
\int d^{4} y \sqrt{g} \psi_{1,1}^{*} \psi_{1,1} & \rightarrow \int d^{4} y h_{2} \psi_{\bar{z}} \psi_{z} \\
\int d^{4} y \sqrt{g} \psi_{1,2}^{*} \psi_{1,2} & \rightarrow \int d^{4} y h_{1} \psi_{\bar{w}} \psi_{w} \\
\int d^{4} y \sqrt{g} \psi_{2,1}^{*} \psi_{2,1} & \rightarrow \int d^{4} y \chi^{*} \chi
\end{aligned}
$$

The terms on the right-hand side are precisely those emerging from the kinetic terms $\int \omega \wedge A \wedge \bar{A}$ and $\int \chi \wedge \bar{\chi}$ in the Lagrangian in the appendix of [2]. In this way we see that the different kinetic terms for matter in the topologically twisted theory all arise from the same term $\int \sqrt{g} \psi^{\dagger} \psi$ in the super Yang-Mills theory.

\subsection{Yukawa couplings}

The Yukawa couplings of the topologically twisted theory can be related to Yang-Mills theory in the same way the equations of motion can. In dimensional reduction, all Yukawa couplings arise from the trilinear interactions in the Yang-Mills Lagrangian. This section will in part follow [29], but will extend and improve the discussion from that paper.

In the super Yang-Mills theory the Yukawa couplings all descend from the terms (dropping overall constants)

$$
\int d^{8} x \sqrt{g}\left[\operatorname{Tr}\left(\bar{\lambda}^{I} \Gamma^{M} A_{M}^{J} \lambda^{K}\right)+\operatorname{Tr}\left(\bar{\lambda}^{I} \Gamma^{r} \phi_{r}^{J} \lambda^{K}\right)\right] .
$$

Here $I, J, K$ are family indices. Both these terms contribute to Yukawa couplings and we consider them separately. Let us start with the second case, where the bosonic field comes from the transverse scalar $\phi^{J}$. In this case

$$
\Gamma^{0} \Gamma^{u}=\left(\begin{array}{ll}
0 & \mathbb{I} \\
\mathbb{I} & 0
\end{array}\right) \otimes\left(\begin{array}{cc}
\mathbb{I} & 0 \\
0 & -\mathbb{I}
\end{array}\right) \otimes\left(\tau^{u}\right),
$$


which gives a chirality flip in both the $0,1,2,3$ and 8,9 directions. To obtain a nonvanishing integral we therefore need $\lambda_{I}$ and $\lambda_{K}$ to be either both of the form $\left(\lambda_{1}+\lambda_{4}\right)$ or both of the form $\left(\lambda_{2}+\lambda_{3}\right)$.

We first assume the form $\lambda_{I}, \lambda_{K}=\left(\lambda_{1}+\lambda_{4}\right)_{I, K}$, when the total Yukawa interaction is

$$
\begin{aligned}
& \mathcal{L}_{\mathrm{YUK}}=\int \sqrt{g} d^{8} x\left(\lambda_{4, I}^{\dagger} \Gamma^{0} \Gamma^{M} A_{M, J} \lambda_{1, K}+\lambda_{1, I}^{\dagger} \Gamma^{0} \Gamma^{M} A_{M, J} \lambda_{4, K}\right)
\end{aligned}
$$

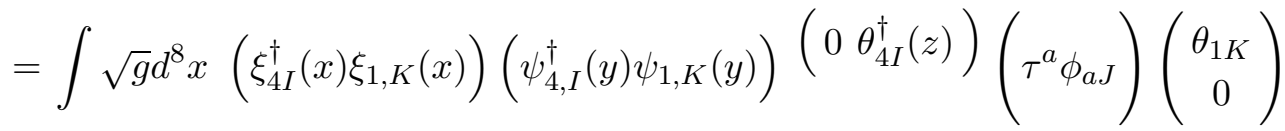

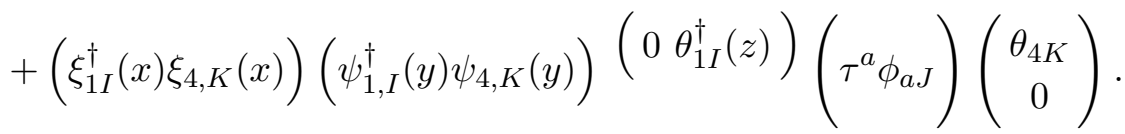

Using the relations (3.4), we can put all expressions in terms of $\lambda_{1}$ alone, eliminating all $\lambda_{4}$ dependence. As $\lambda_{4}$ corresponds to the CPT conjugate of $\lambda_{1}$, by working only with $\lambda_{1}$ we are in effect working only with left-handed spinors, treating these as fundamental as in the usual approach to constructing supersymmetric Lagrangians. We also take $\left(\theta_{1}(z) 0\right)=\left(\begin{array}{ll}1 & 0\end{array}\right)$ as the transverse direction is trivial. We obtain

$$
\begin{aligned}
\mathcal{L}_{\mathrm{YUK}}= & 2 \int d^{4} x\left(\xi_{1 I}^{T}(x) \sigma_{y} \xi_{1 K}(x) \phi_{\bar{u}, J}(x)\right) \int \sqrt{g} d^{4} y\left(\psi_{1 I}^{T}(y) \sigma_{y} \psi_{1 K}(y)\right) \phi_{\bar{u}, J}(y) \\
& +2 \int d^{4} x\left(\xi_{1 I}^{\dagger}(x) \sigma_{y} \xi_{1 K}^{*}(x) \phi_{u, J}(x)\right) \int \sqrt{g} d^{4} y\left(\psi_{1 I}^{\dagger}(y) \sigma_{y} \psi_{1 K}^{*}(y)\right) \phi_{u, J}(y) \\
= & 2 \int d^{4} x\left(\xi_{1 I}^{T}(x) \sigma_{y} \xi_{1 K}(x) \phi_{\bar{u}, J}(x)\right) \int \sqrt{g} d^{4} y\left(\psi_{1 I}^{T}(y) \sigma_{y} \psi_{1 K}(y)\right) \phi_{\bar{u}, J}(y)+\text { c.c }
\end{aligned}
$$

Here $\phi_{u(\bar{u})}(y)=\phi_{8}(y)+(-) i \phi_{9}(y)$ and we have a trivial (flat) metric in the non-compact directions. Now,

$$
\begin{aligned}
\psi_{1, I}^{T} \sigma_{y} \psi_{1, K}(y) \phi_{\bar{u}, J} & =\left(\psi_{1,1}^{I} \psi_{1,2}^{I}\right)\left(\begin{array}{cc}
0 & -i \\
i & 0
\end{array}\right)\left(\begin{array}{c}
\psi_{1,1}^{K} \\
\psi_{1,2}^{K}
\end{array}\right) \\
& =i\left(\psi_{1,2}^{I} \psi_{1,1}^{K}-\psi_{1,1}^{I} \psi_{1,2}^{K}\right) \phi_{\bar{u}, J} .
\end{aligned}
$$

The total interaction is therefore

$$
2 i \int d^{4} x\left(\xi_{1, I}^{T} \sigma_{y} \xi_{1, K} \phi_{\bar{u}, J}\right) \int \sqrt{g} d^{4} y\left(\psi_{1,2}^{I} \psi_{1,1}^{K}-\psi_{1,1}^{I} \psi_{1,2}^{K}\right) \phi_{\bar{u}, J}+\text { c.c }
$$

There is also another possible term, where we let $\lambda^{I}=\left(\lambda_{2}+\lambda_{3}\right)$ and $\lambda^{K}=\left(\lambda_{2}+\lambda_{3}\right)$. However these interactions do not give rise to Yukawa couplings. These couplings always involve a contribution from $\psi_{2,2}$. The mode $\psi_{2,2}$ corresponds to the gaugino that partners the $4 \mathrm{~d}$ gauge boson. Such interactions are therefore gauge rather than Yukawa couplings.

So instead we move on to consider terms coming from the interaction

$$
\operatorname{Tr}\left(\bar{\lambda}^{I} \Gamma^{M}\left[A_{M}^{J}, \lambda^{K}\right]\right),
$$

where we now draw the scalar part of the Yukawa coupling from the $A_{M}$ vector field. In this case

$$
\Gamma^{0} \Gamma^{M}=\left(\begin{array}{ll}
0 & \mathbb{I} \\
\mathbb{I} & 0
\end{array}\right) \otimes\left(\begin{array}{c}
\tilde{\gamma}^{m}
\end{array}\right) \otimes \mathbb{I},
$$


which generates a chirality flip in both the $0,1,2,3$ and 4,5,6,7 directions. In this case to obtain a non-vanishing integral $\lambda_{I}$ and $\lambda_{K}$ must take different forms. There are two options: we first consider the case $\lambda_{K}=\left(\lambda_{1}+\lambda_{4}\right)$, and $\lambda_{I}=\left(\lambda_{2}+\lambda_{3}\right)$, and subsequently analyse the case $\lambda_{K}=\left(\lambda_{2}+\lambda_{3}\right), \lambda_{I}=\left(\lambda_{1}+\lambda_{4}\right)$.

For the first case the total Yukawa interaction is

$$
\begin{aligned}
\mathcal{L}_{\mathrm{YUK}}= & \int d^{8} x \sqrt{g}\left(\lambda_{3, I}^{\dagger} \Gamma^{0} \Gamma^{M} A_{M, J} \lambda_{1, K}+\lambda_{2, I}^{\dagger} \Gamma^{0} \Gamma^{M} A_{M, J} \lambda_{4, K}\right) \\
= & \int d^{8} x \sqrt{g}\left[\left(\xi_{3, I}^{\dagger}(x) \xi_{1, K}(x)\right)\left(0 \psi_{3, I}^{\dagger}(y)\right)\left(\tilde{\gamma}^{M} A_{\mathrm{MJ}}\right)\left(\begin{array}{c}
\psi_{1, K}(y) \\
0
\end{array}\right)\left(\theta_{3, I}^{\dagger}(z) \theta_{1, K}\right)\right. \\
& \left.+\left(\xi_{2, I}^{\dagger}(x) \xi_{4, K}(x)\right)\left(0 \psi_{2, I}^{\dagger}(y)\right)\left(\tilde{\gamma}^{M} A_{\mathrm{MJ}}\right)\left(\begin{array}{c}
\psi_{4, K}(y) \\
0
\end{array}\right)\left(\theta_{2, I}^{\dagger}(z) \theta_{4, K}\right)\right] \cdot(3.43)
\end{aligned}
$$

We again use the relations (3.4) to write everything in terms of $\lambda_{1}$ and $\lambda_{2}$, and take $\left(\theta_{1}(z) 0\right)=\left(\begin{array}{ll}1 & 0\end{array}\right)$. The Yukawa interactions then become

$$
\begin{aligned}
\mathcal{L}_{\mathrm{YUK}}= & -\int d^{4} x\left(\xi_{2 I}^{T}(x) \sigma_{y} \xi_{1 K}(x) A_{J}(x)\right) \int d^{4} y \sqrt{g}\left(0 \psi_{2, I}^{T} \sigma_{y}\right)\left(\tilde{\gamma}^{M} A_{M, J}\right)\left(\begin{array}{c}
\psi_{1, K}(y) \\
0
\end{array}\right) \\
& +\int d^{4} x\left(\xi_{2 I}^{\dagger}(x) \sigma_{y} \xi_{1 K}^{*}(x) A_{J}(x)\right) \int d^{4} y \sqrt{g}\left(0 \psi_{2, I}^{\dagger}\right)\left(\tilde{\gamma}^{M} A_{\mathrm{MJ}}\right)\left(\begin{array}{c}
\sigma_{y} \psi_{1, K}^{*}(y) \\
0
\end{array}\right) .
\end{aligned}
$$

Using the gamma matrices we can now write

$$
\tilde{\gamma}^{M} A_{M}=2\left(\begin{array}{cccc}
0 & 0 & -i A_{z} & A_{\bar{w}} \\
0 & 0 & A_{w} & -i A_{\bar{z}} \\
i A_{\bar{z}} & A_{\bar{w}} & 0 & 0 \\
A_{w} & i A_{z} & 0 & 0
\end{array}\right)
$$

with $\psi_{2}^{T} \sigma_{y}=i\left(\psi_{2,2}-\psi_{2,1}\right)$. So

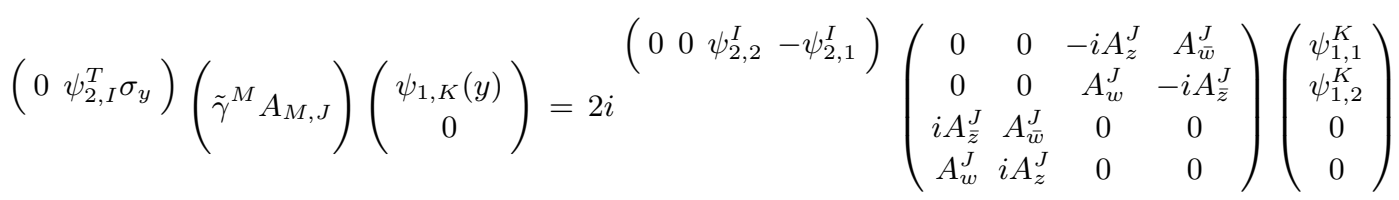

$$
\begin{aligned}
& =2 i\left(-\psi_{2,1}^{I}\left(A_{\bar{w}}^{J} \psi_{1,1}^{k}+i A_{\bar{z}}^{J} \psi_{1,2}^{K}\right)+\psi_{2,2}^{I}\left(i \psi_{1,1}^{K} A_{z}^{J}+A_{w}^{J} \psi_{1,2}^{K}\right)\right) .
\end{aligned}
$$

We also need to evaluate the conjugate expression

$$
\begin{aligned}
\left(\begin{array}{rl}
0 & \psi_{2, I}^{\dagger}
\end{array}\right)\left(\begin{array}{rl}
\tilde{\gamma}^{M} A_{\mathrm{MJ}}
\end{array}\right)\left(\begin{array}{c}
\sigma_{y} \psi_{1, K}^{*}(y) \\
0
\end{array}\right) & =2 i\left(\begin{array}{llll}
0 & 0 & \psi_{2,1}^{I, *} & \psi_{2,2}^{I, *}
\end{array}\right)\left(\begin{array}{cccc}
0 & 0 & -i A_{z}^{J} & A_{\bar{w}}^{J} \\
0 & 0 & A_{w} & -i A_{\bar{z}} \\
i A_{\bar{z}}^{J} & A_{\bar{w}}^{J} & 0 & 0 \\
A_{w}^{J} & i A_{z}^{J} & 0 & 0
\end{array}\right)\left(\begin{array}{c}
-\psi_{1,2}^{K, *} \\
\psi_{1,1}^{K, *} \\
0 \\
0
\end{array}\right) \\
& =2 i\left(-\psi_{2,1}^{I, *}\left(-A_{\bar{w}}^{J} \psi_{1,1}^{K, *}+i A_{\bar{z}}^{J} \psi_{1,2}^{K}\right)+\psi_{2,2}^{I, *}\left(i \psi_{1,1}^{K, *} A_{z}^{J}-A_{w}^{J} \psi_{1,2}^{K, *}\right)\right) .
\end{aligned}
$$

Combining these we evaluate (3.43) as

$$
2 \int d^{8} x \sqrt{g}\left(\xi_{2}^{I, T} \sigma_{y} \xi_{1}^{K}\right)\left(\psi_{2,1}^{I}\left(-A_{z}^{J} \psi_{1,2}^{K}+i A_{w}^{J} \psi_{1,1}^{k}\right)+\psi_{2,2}^{I}\left(-i A_{\bar{w}}^{J} \psi_{1,2}^{K}+\psi_{1,1}^{K} A_{\bar{z}}^{J}\right)\right)+\text { c.c }
$$


The first part of this expression we can interpret as a Yukawa interaction. The second part (involving $\psi_{2,2}$ ) should be interpreted as a gauge interaction as it involves the 4-dimensional gaugino.

Finally we examine the case of $\lambda^{I}=\lambda_{1}+\lambda_{4}$ and $\lambda^{K}=\lambda_{2}+\lambda_{3}$. This gives

$$
\begin{aligned}
\mathcal{L}_{\mathrm{YUK}}= & \int d^{8} x \sqrt{g}\left(\lambda_{4, I}^{\dagger} \Gamma^{0} \Gamma^{M} A_{M, J} \lambda_{2, K}+\lambda_{1, I}^{\dagger} \Gamma^{0} \Gamma^{M} A_{M, J} \lambda_{3, K}\right) \\
= & \int d^{4} x\left(\xi_{1 I}^{T}(x) \sigma_{y} \xi_{2 K}(x)\right) \int d^{4} y \sqrt{g}\left(\psi_{1, I}^{T} \sigma_{y} 0\right)\left(\tilde{\gamma}^{M} A_{\mathrm{MJ}}\right)\left(\begin{array}{c}
0 \\
\psi_{2, K}(y)
\end{array}\right) \\
& -\int d^{4} x\left(\xi_{1 I}^{\dagger}(x) \sigma_{y} \xi_{2 K}^{*}(x)\right) \int d^{4} y \sqrt{g}\left(\psi_{1, I}^{\dagger} 0\right)\left(\tilde{\gamma}^{M} A_{M, J}\right)\left(\begin{array}{c}
0 \\
\sigma_{y} \psi_{2, K}^{*}(y)
\end{array}\right) .
\end{aligned}
$$

We find

$$
\begin{aligned}
& \left(\begin{array}{ll}
\psi_{1, I}^{T} \sigma_{y} & 0
\end{array}\right)\left(\tilde{\gamma}^{M} A_{\mathrm{MJ}}\right)\left(\begin{array}{c}
0 \\
\psi_{2, K}(y)
\end{array}\right)=2 i\left(\psi_{2,1}^{K}\left(-i A_{z}^{J} \psi_{1,2}^{I}-\psi_{1,1}^{I} A_{w}^{J}\right)\right. \\
& \left.+\psi_{2,2}^{K}\left(A_{\bar{w}} \psi_{1,2}^{I}+i A_{\bar{z}}^{J} \psi_{1,1}^{I}\right)\right) \\
& \text { - }\left(\begin{array}{ll}
\psi_{1, I}^{\dagger} \sigma_{y} & 0
\end{array}\right)\left(\tilde{\gamma}^{M} A_{M, J}\right)\left(\begin{array}{c}
0 \\
\sigma_{y} \psi_{2, K}^{*}(y)
\end{array}\right)=-2 i\left(\psi_{2,1}^{K, *}\left(\psi_{1,1}^{I, *} A_{\bar{w}}^{J}-i \psi_{1,2}^{I, *} A_{\bar{z}}^{J}\right)\right. \\
& \left.+\psi_{2,2}^{*}\left(i \psi_{1,1}^{I, *} A_{z}^{J}-\psi_{1,2}^{I, *} A_{w}^{J}\right)\right) .
\end{aligned}
$$

We evaluate (3.46) as

$$
2 \int d^{8} x \sqrt{g}\left(\xi_{1 I}^{T}(x) \sigma_{y} \xi_{2 K}(x)\right)\left(\psi_{2,1}^{K}\left(A_{z}^{J} \psi_{1,2}^{I}-i \psi_{1,1}^{I} A_{w}^{J}\right)+\psi_{2,2}^{K}\left(i A_{\bar{w}} \psi_{1,2}^{I}-A_{\bar{z}}^{J} \psi_{1,1}^{I}\right)\right)+\text { c.c. }
$$

As for (3.41), the first part of this expression should be interpreted as a Yukawa interaction and the second part as a gauge interaction.

Let us gather together the three contributions to Yukawa interactions from (3.41), (3.45) and (3.47). Put together, these give

$$
\begin{aligned}
& 2 \int d^{8} x \sqrt{g}\left(\left(\xi_{1}^{T, I} \sigma_{y} \xi_{1}^{K}\right)\left[i\left(\psi_{1,2}^{I} \psi_{1,1}^{K}-\psi_{1,1}^{I} \psi_{1,2}^{K}\right) \phi_{\bar{u}}^{J}\right]+\right. \\
& \left.\left(\xi_{2}^{T, I} \sigma_{y} \xi_{1}^{K}\right)\left[\psi_{2,1}^{I}\left(-A_{z}^{J} \psi_{1,2}^{K}+i A_{w}^{J} \psi_{1,1}^{K}\right)\right]+\left(\xi_{1}^{T, I} \sigma_{y} \xi_{2}^{K}\right)\left[\psi_{2,1}^{K}\left(A_{z} \psi_{1,2}^{I}-i \psi_{1,1}^{I} A_{w}^{J}\right)\right]\right)+ \text { c.c }
\end{aligned}
$$

There are a total of six different interactions contributing to Yukawa couplings. Using the above dictionary, these Yukawa couplings can be seen to all descend from $A^{I} \wedge A^{J} \wedge \Phi^{k}$ on cyclically permuting indices.

As both the equations of motion, kinetic terms and Yukawa couplings are precisely the same from both dimensional reduction of super Yang-Mills and via the twisted theory, we are free to compute with either. One advantage of the Yang-Mills formalism is that it connects with a set of intuitions that are not as manifestly obvious in the language of the twisted theory. 


\subsection{Holomorphy constraints on the effective theory}

Classical Yang-Mills theory has simple scaling properties and so this suggests that any theory which can be related to Yang-Mills simply by field redefinitions should also possess a similar property, namely that rescalings of the metric can have only a very limited effect on the physical properties of the theory.

Let us recall the equations of motion for the topological theory,

$$
\begin{aligned}
\omega \wedge \partial_{A} \psi+\frac{i}{2}[\bar{\phi}, \chi] & =0, \\
\bar{\partial}_{A} \chi-[\phi, \psi] & =0 .
\end{aligned}
$$

Here $(\phi, \chi)$ are grouped inside the same superfield $\Phi$ as are $(A, \psi)$. The Yukawa couplings are given by expanding $\int A \wedge A \wedge \Phi$ and the kinetic terms come from the terms

$$
\int d^{8} x\left(-2 \omega \wedge D_{\mu} \bar{\psi} \wedge \bar{\sigma}^{\mu} \psi\right), \quad \int d^{8} x\left(D_{\mu} \chi \wedge \sigma^{\mu} \bar{\chi}\right)
$$

$\phi$ can attain an arbitrary holomorphic vev within the Cartan subalgebra. We suppose

$$
\langle\phi\rangle=f\left(z_{1}, z_{2}\right) t^{1}+g\left(z_{1}, z_{2}\right) t^{2}
$$

with the matter curves located at $f=0, g=0$ and $f+g=0$.

There is one important and simple set of solutions to (3.48), given by the rescalings

$$
\begin{aligned}
\phi & \rightarrow \lambda \phi, \\
\chi & \rightarrow \lambda \chi, \\
\omega & \rightarrow \lambda^{2} \chi, \\
(A, \psi) & \rightarrow(A, \psi) .
\end{aligned}
$$

It is manifest that given a set of solutions to (3.48) this rescaling provides a new set of solutions. The rescalings of (3.49) correspond to the overall metric rescaling $g_{i j} \rightarrow \lambda^{2} g_{i j}$. To see this, note first that this rescales the Kähler form as in (3.49). The scaling of $\phi$ is most easily seen by considering the type IIB case, where $\phi$ can be interpreted as the transverse separation of the brane stacks. Such a transverse separation grows linearly with the overall length scale consistent with (3.49). $\chi$ belongs in the same supermultiplet as $\phi$ and so scales in the same fashion.

$A$ does not rescale, and so the unnormalised Yukawa couplings scale as

$$
\int A \wedge A \wedge \Phi \longrightarrow \lambda \int A \wedge A \wedge \Phi
$$

as every contributing term has one factor of $\chi$. The kinetic terms scale as

$$
\int \omega \wedge D_{\mu} \bar{\psi} \wedge \sigma^{\mu} \psi \rightarrow \lambda^{2} \int \omega \wedge D_{\mu} \bar{\psi} \wedge \sigma^{\mu} \psi, \quad \int D_{\mu} \chi \wedge \sigma^{\mu} \bar{\chi} \rightarrow \lambda^{2} \int D_{\mu} \chi \wedge \sigma^{\mu} \bar{\chi}
$$

and so all kinetic terms scale as $Z \rightarrow \lambda^{2} Z$. 
The normalised (physical) Yukawa couplings therefore scale as

$$
\frac{\int A^{a} \wedge A^{b} \wedge \Phi^{c}}{\sqrt{Z_{a} Z_{b} Z_{c}}} \rightarrow \frac{\lambda \int A^{a} \wedge A^{b} \wedge \Phi^{c}}{\sqrt{\left(\lambda^{2} Z_{a}\right)\left(\lambda^{2} Z_{b}\right)\left(\lambda^{2} Z_{c}\right)}}=\frac{1}{\lambda^{2}} \frac{\int A^{a} \wedge A^{b} \wedge \Phi^{c}}{\sqrt{Z_{a} Z_{b} Z_{c}}} .
$$

The metric rescaling increases the size of the 4-cycle on which the 7-branes supporting the Yang-Mills theory live, and therefore rescales $\alpha_{\mathrm{GUT}}^{-1} \rightarrow \lambda^{4} \alpha_{\mathrm{GUT}}^{-1}$. Physical Yukawa couplings therefore behave as $Y_{\alpha \beta \gamma}^{\text {phys }} \sim \alpha_{\mathrm{GUT}}^{1 / 2}$, with $\alpha_{\mathrm{GUT}}$ appearing as a universal prefactor.

This conclusion could have been anticipated based on holomorphy and the structure of 4-dimensional supergravity theories. These provide very powerful general constraints on the structure of the effective action. The gauge coupling $\alpha^{-1}$ appears in the action as the coefficient of the kinetic term $F_{\mu \nu} F^{\mu \nu}$. In string theory the gauge coupling is dynamical, and so is part of a Kähler modulus ${ }^{1} T_{\mathrm{GUT}}=\frac{4 \pi}{g^{2}}+i a$, whose imaginary part is an axion carrying a perturbative shift symmetry.

As the imaginary part is axionic there is a perturbative shift symmetry $T \rightarrow T+i \epsilon$, which implies that $T$ can make no perturbative appearance in the superpotential. Consequently the superpotential Yukawa couplings cannot depend on $\alpha_{\mathrm{GUT}}$, which only enters the Yukawa couplings via prefactors derived from the Kähler potential.

The absence of $\alpha_{\mathrm{GUT}}$ from the superpotential is a specific example of a general phenomenon: as all Kähler moduli have shift symmetries which are exact within perturbation theory, the superpotential cannot depend on Kähler moduli. However, from an effective field theory viewpoint the superpotential can have arbitrary dependence on complex structure moduli and there is no reason not to expect a rank 3 Yukawa matrix.

\section{Wavefunctions and Yukawas with background fluxes}

In this section we explicitly solve the equations of motion to obtain the matter wavefunctions in the presence of background flux. In section 4.1 we solve the D-term equations which require a perturbed background metric and discuss the perturbative expansion used in finding the wavefunction solutions. Subsequently in sections 4.2 and 4.3 we present solutions to the equations of motion. In section 4.4 we use the wavefunction profiles to calculate the resulting Yukawa couplings, finding no corrections to a rank-1 matrix.

\subsection{Flux solutions to D-terms}

We first set out the background flux configuration. We consider only Abelian flux and so there are three relevant generators for a rank 2 enhancement point. We denote these $Q_{0}$, $Q_{1}$ and $Q_{2}$, with $Q_{0}$ corresponding to the hypercharge flux. We denote the corresponding flux and gauge fields with the index $I$ such that

$$
A=\sum_{I} A_{I}=A_{0}+A_{1}+A_{2}
$$

The constraints on the flux from the equations of motion are

$$
F^{(2,0)}=F^{(0,2)}=0 .
$$

\footnotetext{
${ }^{1}$ We restrict to language appropriate to IIB/F-theory compactifications.
} 
We also have the D-term

$$
\omega \wedge F^{(1,1)}=0 .
$$

In this paper we consider the factorised case with no $d z_{1} \wedge d \bar{z}_{\overline{2}}$ terms

$$
F=F_{1 \overline{1}} d z_{1} \wedge d \bar{z}_{\overline{1}}+F_{2 \overline{2}} d z_{2} \wedge d \bar{z}_{\overline{2}} .
$$

Apart from simplicity, cross terms in the flux do not affect the D-term equation for a metric ansatz (2.14) and so we do not expect new effects regarding their compatibility with supersymmetry. We take the expansion to first order as in [27] and also define $\tilde{F}$ through

$$
\begin{aligned}
& F_{1 \overline{1}}=2 i M+4 i\left(\alpha_{1} z_{1}+\bar{\alpha}_{1} \bar{z}_{\overline{1}}\right) \equiv 2 i M+i \tilde{F}_{1 \overline{1}} \\
& F_{2 \overline{2}}=2 i N+4 i\left(\alpha_{2} z_{2}+\bar{\alpha}_{2} \bar{z}_{\overline{2}}\right) \equiv 2 i N+i \tilde{F}_{2 \overline{2}} .
\end{aligned}
$$

Here $M, N, \alpha_{i}, \tilde{F}$ all have a suppressed generator index $I$. Next we perform a gauge transformation as in [27]

$$
\hat{A}=A-d \Omega
$$

such that $\hat{A}_{\overline{1}}=\hat{A}_{\overline{2}}=0$. The corresponding potentials read

$$
\begin{aligned}
& \hat{A}_{1}=-2 i M \bar{z}_{\overline{1}}-2 i\left(\bar{\alpha}_{1} \bar{z}_{\overline{1}}^{2}+2 \alpha_{1} z_{1} \bar{z}_{\overline{1}}\right), \\
& \hat{A}_{2}=-2 i N \bar{z}_{\overline{2}}-2 i\left(\bar{\alpha}_{2} \bar{z}_{\overline{2}}^{2}+2 \alpha_{2} z_{2} \bar{z}_{\overline{2}}\right) .
\end{aligned}
$$

Taking the Kahler form as

$$
\omega=\frac{i}{2}\left(1+f_{1}\right) d z_{1} \wedge d \bar{z}_{\overline{1}}+\frac{i}{2}\left(1+f_{2}\right) d z_{2} \wedge d \bar{z}_{\overline{2}},
$$

the D-term is solved by

$$
M=-N, \quad f_{1}=-\frac{\tilde{F}_{1 \overline{1}}}{2 N}, \quad f_{2}=\frac{\tilde{F}_{2 \overline{2}}}{2 N} .
$$

This fixes the Kahler form, up to an overall constant rescaling. ${ }^{2}$ It is important to note that the D-terms require a non-trivial metric background. Note that $f_{1}$ and $f_{2}$ are $O(1)$ in the flux, which will imply that the leading metric-induced perturbations to the wavefunctions dominates over the flux-induced perturbations. In turn this will mean that the wavefunction solutions that we find will be different to those presented in [27].

There are three copies of the equations (4.10) due to the generator index $I$. This then implies

$$
\frac{\alpha_{i, I}}{N_{I}}=\frac{\alpha_{i, J}}{N_{J}} \equiv n_{i} \quad \forall I, J
$$

where we define $n_{i}$ through

$$
\begin{aligned}
& f_{1}=-2\left(n_{1} z_{1}+\bar{n}_{\overline{1}} \bar{z}_{\overline{1}}\right), \\
& f_{2}=2\left(n_{2} z_{2}+\bar{n}_{\overline{2}} \bar{z}_{\overline{2}}\right) .
\end{aligned}
$$

\footnotetext{
${ }^{2}$ Note that the Bianchi identity for the flux ensures the Kahler condition on the metric.
} 
We now give a vev to $\varphi$ such that

$$
\langle\varphi\rangle=\frac{z_{1}}{v} Q_{1}+\frac{z_{2}}{v} Q_{2} .
$$

The equations of motion to be solved then read

$$
\begin{aligned}
& \left(1+f_{1}\right)\left(\partial_{2}-i q \cdot \hat{A}_{2}\right) \hat{\psi}_{\overline{2}}+\left(1+f_{2}\right)\left(\partial_{1}-i q \cdot \hat{A}_{1}\right) \hat{\psi}_{\overline{1}}-\frac{1}{v}\left(\bar{z}_{1} q_{1}+\bar{z}_{2} q_{2}\right) \hat{\chi}=0, \\
& \bar{\partial}_{\overline{1}} \hat{\chi}-\frac{1}{v}\left(z_{1} q_{1}+z_{2} q_{2}\right) \hat{\psi}_{\overline{1}}=0 \text {, } \\
& \bar{\partial}_{\overline{2}} \hat{\chi}-\frac{1}{v}\left(z_{1} q_{1}+z_{2} q_{2}\right) \hat{\psi}_{\overline{2}}=0 \text {. }
\end{aligned}
$$

Here $q$ - denotes contraction into the vector of charges $q=\left(q_{0}, q_{1}, q_{2}\right)$.

In order to solve for the wavefunctions we perform a perturbative expansion. In [10] the relevant expansions were identified as the derivative expansion, which is an expansion in $v^{1 / 2}$, and the flux expansion, which is an expansion in $\alpha_{i} v^{3 / 2}$. Which one dominated depended on the size of the flux parameters $\alpha_{i}$ and their higher order analogues. In our case the appropriate expansions are in the metric deformations as these dominate over the flux contributions to the wavefunctions. To see this note that the flux contributions to the wavefunctions come in at $O\left(\alpha_{i} v^{3 / 2}\right)$ [10,27], while using the solutions (4.21) we see that the leading contribution from the metric expansion comes in at $O\left(n_{i} v^{1 / 2}\right)$. This is leading in powers of $v$ but also note that $n_{i} \gg \alpha_{i}$ since $M \ll 1$. In the coming sections we solve the wavefunctions to second order in the 'metric' expansion. ${ }^{3}$

\subsection{Matter wavefunctions}

The matter curves correspond to the curves $z_{1}=0$ and $z_{2}=0$. By symmetry it is sufficient to solve for one of the curves which in this case we take to be the $z_{1}=0$ curve. In terms of the charges defined in section 4.1 the curve corresponds to

$$
\left(q_{0}, q_{1}, q_{2}\right)=\left(q_{0}, 1,0\right)
$$

Since we are only solving the equations of motion to $\mathcal{O}\left(z_{i}\right)=\mathcal{O}\left(v^{1 / 2}\right)^{4}$ we can drop the flux terms and so the equations of motion read

$$
\begin{aligned}
\left(1+f_{1}\right) \partial_{2} \psi_{\overline{2}}+\left(1+f_{2}\right) \partial_{1} \psi_{\overline{1}}-\frac{\bar{z}_{1}}{v} \chi & =0, \\
\bar{\partial}_{\overline{1}} \chi-\frac{z_{1}}{v} \psi_{\overline{1}} & =0, \\
\bar{\partial}_{\overline{2}} \chi-\frac{z_{1}}{v} \psi_{\overline{2}} & =0
\end{aligned}
$$

The equations (4.17) and (4.18) are trivially solved exactly for $\psi_{\overline{1}}$ and $\psi_{\overline{2}}$ in terms of $\chi$.

\footnotetext{
${ }^{3}$ We only calculate the contributions from the fluxes (4.6), i.e. we do not consider higher order terms such as $\beta_{i} z_{1}^{2}$. For the first order solutions this does not matter since they are subleading, but they could possibly compete at second order. This is highly dependent on the choice for the parameters and so for simplicity we drop such terms.

${ }^{4}$ Note that a wavefunction solution at $\mathcal{O}\left(z_{i}^{r}\right)$, for some $r$, solves the equations of motion to $\mathcal{O}\left(z_{i}^{r-1}\right)$.
} 
The perturbative solutions are based on the zeroth order solution, obtained by taking $f_{1}=f_{2}=0$, which we reproduce here for convenience

$$
\chi^{(0)}=g\left(z_{2}\right) e^{-\frac{\left|z_{1}\right|^{2}}{v}} .
$$

Here $g$ is a holomorphic function of $z_{2}$ which carries a suppressed generation index $g_{a}\left(z_{2}\right)=$ $\left\{1, z_{2}, z_{2}^{2}\right\}$. The full set of equations are solved to $\mathcal{O}(z)$ by the $\mathcal{O}\left(z^{2}\right)$ solution

$$
\begin{aligned}
\chi= & \chi^{(0)}\left\{1+\frac{\left|z_{1}\right|^{2}}{v}\left[\frac{f_{2}}{2}+\frac{1}{2} v \bar{n}_{\overline{2}}\left(\partial_{2} \ln g\right)-\frac{3 f_{2}^{2}}{8}-\frac{5}{4} v\left|n_{2}\right|^{2}-v \bar{n}_{\overline{2}} f_{2}\left(\partial_{2} \ln g\right)\right.\right. \\
& \left.-\frac{7}{8} v^{2} \bar{n}_{\overline{2}}^{2}\left(\left(\partial_{2} \partial_{2} \ln g\right)+\left(\partial_{2} \ln g\right)^{2}\right)\right]+\frac{\left|z_{1}\right|^{4}}{v^{2}}\left[\frac{f_{2}^{2}}{8}+\frac{1}{4} v\left|n_{2}\right|^{2}+\frac{1}{4} v \bar{n}_{\overline{2}} f_{2}\left(\partial_{2} \ln g\right)\right. \\
& \left.\left.+\frac{1}{8} v^{2} \bar{n}_{\overline{2}}^{2}\left(\left(\partial_{2} \partial_{2} \ln g\right)+\left(\partial_{2} \ln g\right)^{2}\right)\right]+\frac{\bar{n}_{2}}{3}\left(\partial_{2} \ln g\right) f_{1}\left|z_{1}\right|^{2}-\frac{2 v}{3} z_{1} n_{1} \bar{n}_{2}\left(\partial_{2} \ln g\right)\right\} .
\end{aligned}
$$

The solution for the second matter curve $z_{2}=0$ is obtained from (4.21) by the operations

$$
z_{1} \leftrightarrow z_{2}, n_{1} \leftrightarrow-n_{2} \quad\left(\Longrightarrow f_{1} \leftrightarrow f_{2}\right)
$$

\subsection{Higgs wavefunctions}

The Higgs curve is the curve $z_{1}+z_{2}=0$ which corresponds to charges

$$
\left(q_{0}, q_{1}, q_{2}\right)=\left(q_{0},-1,-1\right) .
$$

The order counting of the derivative expansion treats $z_{1}$ and $z_{2}$ on an equal footing and so applies unaltered to this solution. Again we solve the equations of motion to $\mathcal{O}(z)$ obtaining a solution for $\chi$ to $\mathcal{O}\left(z^{2}\right)$. The flux terms in the equations of motion only come in at higher orders and so can be dropped.

As suggested in [27], to find the solutions it is convenient to define

$$
\begin{aligned}
w & \equiv z_{1}+z_{2}, \quad u \equiv z_{1}-z_{2}, \\
\psi_{\bar{w}} & \equiv \frac{1}{2}\left(\psi_{\overline{1}}+\psi_{\overline{2}}\right), \quad \psi_{\bar{u}} \equiv \frac{1}{2}\left(\psi_{\overline{1}}-\psi_{\overline{2}}\right), \\
n_{+} & \equiv \frac{1}{2}\left(n_{1}+n_{2}\right), \quad n_{-} \equiv \frac{1}{2}\left(n_{1}-n_{2}\right), \\
f_{+} & \equiv-\frac{1}{2}\left(f_{1}+f_{2}\right)=n_{+} u+n_{-} w+\bar{n}_{+} \bar{u}+\bar{n}_{-} \bar{w}, \\
f_{-} & \equiv \frac{1}{2}\left(f_{2}-f_{1}\right)=n_{+} w+n_{-} u+\bar{n}_{+} \bar{w}+\bar{n}_{-} \bar{u} .
\end{aligned}
$$

With this change of variables the equations of motion read

$$
\begin{aligned}
\left(1-f_{+}\right)\left(\partial_{w} \psi_{\bar{w}}+\partial_{u} \psi_{\bar{u}}\right)+f_{-}\left(\partial_{w} \psi_{\bar{u}}+\partial_{u} \psi_{\bar{w}}\right)+\frac{\bar{w}}{2 v} \hat{\chi} & =0, \\
\bar{\partial}_{\bar{w}} \chi+\frac{w}{v} \psi_{\bar{w}} & =0, \\
\bar{\partial}_{\bar{u}} \chi+\frac{w}{v} \psi_{\bar{u}} & =0 .
\end{aligned}
$$


The perturbative expansion is based on a zeroth order solution which solves the equations of motion for $f_{+}=f_{-}=0$ and reads $[27]$

$$
\chi^{(0)}=e^{-\frac{|w|^{2}}{\sqrt{2} v}}, \psi_{\bar{w}}^{(0)}=\frac{1}{\sqrt{2}} \chi^{(0)}, \psi_{\bar{u}}^{(0)}=0 .
$$

As we regard this as a Higgs curve, we are only concerned with a single solution so that the holomorphic function is just taken to be a constant.

The equations of motion are solved to $\mathcal{O}(z)$ by the $\mathcal{O}\left(z^{2}\right)$ expression

$$
\begin{aligned}
\chi= & \chi^{(0)}\left[1-\frac{1}{3 \sqrt{2} v}\left(|w|^{2}\left(\frac{3}{2} n_{+} u+\frac{3}{2} \bar{n}_{+} \bar{u}+n_{-} w+\bar{n}_{-} \bar{w}\right)+\sqrt{2} v n_{-} w\right)+\chi^{(2)}\right], \\
\chi^{(2)}= & w\left(\varphi_{1}+u \varphi_{2}+\bar{u} \varphi_{3}+|u|^{2} \varphi_{4}+u^{2} \varphi_{5}+\bar{u}^{2} \varphi_{6}\right), \\
\varphi_{1}= & \frac{n_{-}^{2}}{36 v^{2}} w|w|^{4}+\frac{\left|n_{-}\right|^{2}}{18 v^{2}} \bar{w}|w|^{4}+\frac{\bar{n}_{-}^{2}}{36 v^{2}} \bar{w}^{3}|w|^{2}+\frac{\sqrt{2}}{8 v} \bar{w}|w|^{2}\left(\frac{5\left|n_{+}\right|^{2}}{4}-\left|n_{-}\right|^{2}\right) \\
& +\left(-\frac{\sqrt{2} n_{-}^{2}}{24 v}+\frac{\sqrt{2} n_{+}^{2}}{16 v}\right) w|w|^{2}-\frac{\sqrt{2}}{8 v} \bar{w}^{3}\left(\frac{7 \bar{n}_{-}^{2}}{9}-\frac{\bar{n}_{+}^{2}}{2}\right)+\bar{w}\left(-\frac{5}{16}\left|n_{+}\right|^{2}-\frac{\left|n_{-}\right|^{2}}{4}\right) \\
& -\left(\frac{n_{-}^{2}}{12}+\frac{n_{+}^{2}}{8}\right) w, \\
\varphi_{2}= & \frac{1}{12 v^{2}}|w|^{4} n_{+} n_{-}+\frac{1}{12 v^{2}} \bar{w}^{2}|w|^{2} n_{+} \bar{n}_{-}-\frac{\sqrt{2}}{12 v}|w|^{2} n_{+} n_{-} \\
& -\frac{\sqrt{2}}{4 v} \bar{w}^{2}\left(n_{+} \bar{n}_{-}-\frac{1}{3} n_{-} \bar{n}_{+}\right)-\frac{2}{3} n_{+} n_{-}, \\
\varphi_{3}= & \frac{1}{12 v^{2}}|w|^{4} \bar{n}_{+} n_{-}+\frac{1}{12 v^{2}} \bar{w}^{2}|w|^{2} \bar{n}_{+} \bar{n}_{-}-\frac{\sqrt{2}}{12 v}|w|^{2}\left(2 \bar{n}_{+} n_{-}-n_{+} \bar{n}_{-}\right)-\frac{\sqrt{2}}{6 v} \bar{w}^{2} \bar{n}_{+} \bar{n}_{-} \\
& -\frac{1}{3}\left(n_{+} \bar{n}_{-}+n_{-} \bar{n}_{+}\right) \\
\varphi_{4}= & \frac{\left|n_{+}\right|^{2}}{8 v^{2}} \bar{w}|w|^{2}-\frac{3 \sqrt{2}}{8 v} \bar{w}\left|n_{+}\right|^{2}, \\
\varphi_{5}= & \frac{n_{+}^{2}}{16 v^{2}} \bar{w}|w|^{2}-\frac{3 \sqrt{2} n_{+}^{2}}{16 v} \bar{w}, \\
\varphi_{6}= & \frac{\bar{n}_{+}^{2}}{16 v^{2}} \bar{w}|w|^{2}-\frac{3 \sqrt{2} \bar{n}_{+}^{2}}{16 v} \bar{w} .
\end{aligned}
$$

Like the matter curve solutions this solution involves $\bar{z}_{1}$ and $\bar{z}_{2}$ up to second order.

\subsection{Yukawa couplings}

Having calculated the perturbative wavefunctions it is possible to calculate the Yukawa couplings. The computation of the physical Yukawa couplings requires both the holomorphic Yukawa couplings and the normalisation of the kinetic terms.

The kinetic terms originate from terms in the $8 \mathrm{~d}$ Lagrangian

$$
\int d^{8} x-2 \omega \wedge D_{\mu} \bar{\psi} \wedge \bar{\sigma}^{\mu} \psi, \quad \int d^{8} x D_{\mu} \chi \wedge \sigma^{\mu} \bar{\chi}
$$


The contributions of these two terms should be combined to work out the normalisation for the actual zero mode. Evaluation of the kinetic terms is not possible in a purely local framework, as it requires knowledge of the global structure of the zero modes and in particular the full (compact) matter curve along which the zero mode is supported. However it is important to note that the corrected wavefunctions induce inter-generational kinetic mixing since the corrections come in with powers of both $\bar{z}_{\overline{1}}$ and $\bar{z}_{\overline{2}} .{ }^{5}$ Therefore to extract the physical Yukawa couplings requires a diagonalisation of the kinetic terms. The rank of the Yukawa matrix is unaltered by the kinetic terms and so we can analyse this without reference to the kinetic terms.

The holomorphic Yukawas that appear in the superpotential are given by (2.30). In [10] a Frogatt-Nielsen-like mechanism was proposed for generating the observed mass hierarchies. The observation is that the integration volume form respects two symmetries $\mathrm{U}(1)_{1} \times \mathrm{U}(1)_{2}$ given by

$$
z_{1} \rightarrow e^{i \theta_{1}} z_{1}, z_{2} \rightarrow e^{i \theta_{2}} z_{2}
$$

The exponential part of the matter wavefunctions respects these symmetries and the exponential part of the Higgs wavefunctions breaks the symmetry down to the diagonal U(1) given by

$$
\left(z_{1}+z_{2}\right) \rightarrow e^{i \theta}\left(z_{1}+z_{2}\right)
$$

It is this $\mathrm{U}(1)$ that acts as a Frogatt-Nielsen-like charge since any integrals that do not respect it will vanish. This means that the non-exponential parts of the zero mode wavefunctions should combine in a U(1) neutral way. For the top Yukawa coupling this occurs already at tree level. However since the other generations have non-trivial holomorphic functions $g\left(z_{2}, z_{1}\right)$ factors they can only form U(1) neutral combinations through higher order non-holomorphic corrections to the wavefunctions. The idea is that since these are suppressed, they naturally induce a hierarchy.

If such a hierarchy is present, we should be able to find it using the explicit solutions for the wavefunctions presented in sections 4.2 and 4.3. Note that since we have only expanded the wavefunctions to second order in the derivative expansion we can only calculate the leading contributions to the Yukawa couplings $Y_{11}, Y_{12}, Y_{21}, Y_{22}, Y_{31}$ and $Y_{13}$.

The natural variables for calculating the Yukawas are $w$ and $u$ as introduced in eq. (4.23). The non vanishing integrals all take the form

$$
\begin{aligned}
I_{m n} & =\int d^{2} z_{1} d^{2} z_{2} e^{-\frac{1}{v}\left(\left|z_{1}\right|^{2}+\left|z_{2}\right|^{2}+\frac{1}{\sqrt{2}}\left|z_{1}+z_{2}\right|^{2}\right)}|w|^{2 m}|u|^{2 n} \\
& =\int \frac{1}{4} d^{2} w d^{2} u e^{-\frac{1}{v}\left(\frac{1}{2}|u|^{2}+\frac{1}{2 s}|w|^{2}\right)}|w|^{2 m}|u|^{2 n} \\
& =\pi^{2} n ! m !(2 v)^{(n+m+2)} s^{(m+1)}
\end{aligned}
$$

where we define $s=\sqrt{2}-1$. Then calculating the integrals (2.30) simply amounts to extracting the appropriate coefficient multiplying each $I_{m n}$ and summing the result. Such a calculation is naturally done using Mathematica. Performing this calculation we find the

\footnotetext{
${ }^{5}$ This is in contrast to the flux corrected wavefunctions of [27].
} 
following result

$$
\begin{aligned}
& Y_{11}=-4 a \pi^{2} v^{2}, \\
& Y_{i j}=0 \quad \forall(i, j) \neq(1,1) .
\end{aligned}
$$

We therefore find that flux and metric corrected wavefunctions do not induce new Yukawa couplings and the Yukawa matrix remains as rank one.

This is a rather striking result given the form of the perturbed wavefunctions. It hints at a symmetry underlying the vanishing of the Yukawas. In a recent paper [30] precisely such a symmetry principle was shown. The idea is that the gauge symmetry of the 8D super-Yang-Mills can be used to define a kind of generalised cohomology where wavefunctions that differ by a gauge transformation are identified under the cohomology. A representative of each cohomology class was shown to be simply the wavefunctions evaluated on the localisation curve. These are just the holomorphic $g_{i}$ of (4.19). Finally the Yukawa couplings were shown to depend just on the cohomology representatives and so could be evaluated simply by using the $g_{i}$. This directly gives our result (4.33). Therefore (4.33) is a special case of a more general phenomenon for all wavefunctions that are induced by corrections due to gauge flux.

\section{Summary and discussion}

In this article we have studied the inter-relationship of flavour and supersymmetry in the context of F-theory GUTs. In the earlier part of the article we described the relationship between the topologically twisted theory of [2] and the canonical formulation of $8 \mathrm{~d}$ super Yang-Mills theory, deriving the zero mode equations of motion from the latter. We also provided a dictionary between the two formalisms. The simple scaling properties of super Yang-Mills make it clear that metric rescalings (which include scalings of $\alpha_{\mathrm{GUT}}$ ) should not alter the rank of the Yukawa matrix. This is also required by holomorphy consideration of the $4 \mathrm{~d}$ effective supergravity theory.

For metric and flux backgrounds we also solved the equations of motion up to second order in the perturbations. We found that the induced metric deformations are the leading corrections to the wavefunctions dominating over the flux terms. Using the explicit form for the wavefunctions in the vicinity of the triple intersection point we computed the unnormalised Yukawa couplings. We found that the wavefunction deformations did not affect the rank of the Yukawa matrix, which remained rank one. The fundamental reason for this has recently been explained in [30]. The explicit form of the wavefunctions is however important for non-holomorphic properties of the effective theory.

\section{Acknowledgments}

We thank James Gray, Andre Lukas, Anshuman Maharana, Fernando Quevedo and Martijn Wijnholt for helpful discussions. JC is supported by a Royal Society University Research Fellowship. EP is supported in part by the European ERC Advanced Grant 226371 MassTeV, by the CNRS PICS no. 3059 and 4172, by the grants ANR-05-BLAN-0079-02, the PITN contract PITN-GA-2009-237920 and was supported for a substantial part of the work by an STFC Postdoctoral Fellowship. 
Open Access. This article is distributed under the terms of the Creative Commons Attribution Noncommercial License which permits any noncommercial use, distribution, and reproduction in any medium, provided the original author(s) and source are credited.

\section{References}

[1] R. Donagi and M. Wijnholt, Model building with F-theory, arXiv:0802.2969 [SPIRES].

[2] C. Beasley, J.J. Heckman and C. Vafa, GUTs and exceptional branes in F-theory - I, JHEP 01 (2009) 058 [arXiv:0802.3391] [SPIRES].

[3] H. Hayashi, R. Tatar, Y. Toda, T. Watari and M. Yamazaki, New aspects of heterotic-F-theory duality, Nucl. Phys. B 806 (2009) 224 [arXiv:0805.1057] [SPIRES].

[4] L. Aparicio, D.G. Cerdeno and L.E. Ibáñez, Modulus-dominated SUSY-breaking soft terms in F-theory and their test at LHC, JHEP 07 (2008) 099 [arXiv: 0805. 2943] [SPIRES].

[5] C. Beasley, J.J. Heckman and C. Vafa, GUTs and exceptional branes in F-theory - II: experimental predictions, JHEP 01 (2009) 059 [arXiv:0806.0102] [SPIRES].

[6] R. Donagi and M. Wijnholt, Breaking GUT groups in F-theory, arXiv:0808.2223 [SPIRES].

[7] J.J. Heckman and C. Vafa, F-theory, GUTs and the weak scale, JHEP 09 (2009) 079 [arXiv: 0809.1098] [SPIRES].

[8] R. Blumenhagen, V. Braun, T.W. Grimm and T. Weigand, GUTs in type IIB orientifold compactifications, Nucl. Phys. B $\mathbf{8 1 5}$ (2009) 1 [arXiv:0811.2936] [SPIRES].

[9] A. Font and L.E. Ibáñez, Yukawa Structure from U(1) fluxes in F-theory grand unification, JHEP 02 (2009) 016 [arXiv: 0811.2157] [SPIRES].

[10] J.J. Heckman and C. Vafa, Flavor hierarchy from F-theory, arXiv:0811.2417 [SPIRES].

[11] R. Blumenhagen, Gauge coupling unification in F-theory grand unified theories, Phys. Rev. Lett. 102 (2009) 071601 [arXiv:0812.0248] [SPIRES].

[12] J.L. Bourjaily, Local models in F-theory and M-theory with three generations, arXiv: 0901.3785 [SPIRES].

[13] H. Hayashi, T. Kawano, R. Tatar and T. Watari, Codimension-3 singularities and Yukawa couplings in F-theory, Nucl. Phys. B 823 (2009) 47 [arXiv:0901.4941] [SPIRES].

[14] B. Andreas and G. Curio, From local to global in F-theory model building, arXiv:0902.4143 [SPIRES].

[15] C.-M. Chen and Y.-C. Chung, A note on local GUT models in F-theory, Nucl. Phys. B 824 (2010) 273 [arXiv:0903.3009] [SPIRES].

[16] R. Donagi and M. Wijnholt, Higgs bundles and UV completion in F-theory, arXiv: 0904.1218 [SPIRES].

[17] V. Bouchard, J.J. Heckman, J. Seo and C. Vafa, F-theory and neutrinos: Kaluza-Klein dilution of flavor hierarchy, arXiv:0904.1419 [SPIRES].

[18] L. Randall and D. Simmons-Duffin, Quark and lepton flavor physics from F-theory, arXiv:0904.1584 [SPIRES].

[19] J.J. Heckman and C. Vafa, CP Violation and F-theory GUTs, arXiv:0904.3101 [SPIRES]. 
[20] J. Marsano, N. Saulina and S. Schäfer-Nameki, F-theory compactifications for supersymmetric GUTs, JHEP 08 (2009) 030 [arXiv:0904.3932] [SPIRES].

[21] R. Tatar, Y. Tsuchiya and T. Watari, Right-handed neutrinos in F-theory compactifications, Nucl. Phys. B 823 (2009) 1 [arXiv: 0905.2289] [SPIRES].

[22] R. Blumenhagen, T.W. Grimm, B. Jurke and T. Weigand, F-theory uplifts and GUTs, JHEP 09 (2009) 053 [arXiv: 0906.0013] [SPIRES].

[23] J. Marsano, N. Saulina and S. Schäfer-Nameki, Monodromies, fluxes and compact three-generation F-theory GUTs, JHEP 08 (2009) 046 [arXiv:0906.4672] [SPIRES].

[24] J.J. Heckman, A. Tavanfar and C. Vafa, The point of $E_{8}$ in F-theory GUTs, arXiv:0906.0581 [SPIRES].

[25] R. Blumenhagen, J.P. Conlon, S. Krippendorf, S. Moster and F. Quevedo, SUSY breaking in local string/F-theory models, JHEP 09 (2009) 007 [arXiv: 0906. 3297] [SPIRES].

[26] J.P. Conlon and E. Palti, On Gauge Threshold Corrections for Local IIB/F-theory GUTs, Phys. Rev. D 80 (2009) 106004 [arXiv:0907.1362] [SPIRES].

[27] A. Font and L.E. Ibáñez, Matter wave functions and Yukawa couplings in F-theory grand unification, JHEP 09 (2009) 036 [arXiv: 0907.4895] [SPIRES].

[28] R. Blumenhagen, T.W. Grimm, B. Jurke and T. Weigand, Global F-theory GUTs, arXiv:0908.1784 [SPIRES].

[29] J.P. Conlon, A. Maharana and F. Quevedo, Wave functions and Yukawa couplings in local string compactifications, JHEP 09 (2008) 104 [arXiv: 0807.0789] [SPIRES].

[30] S. Cecotti, M.C.N. Cheng, J.J. Heckman and C. Vafa, Yukawa couplings in F-theory and non-commutative geometry, arXiv:0910.0477 [SPIRES]. 